\title{
THE RIEMANN PROBLEM FOR GENERAL $2 \times 2$ CONSERVATION LAWS
}

BY

\section{TAI-PING LIU( $\left.{ }^{(}\right)$}

ABSTRACT. The Riemann Problem for a system of hyperbolic conservation laws of form

$$
\begin{aligned}
& u_{t}+f(u, v)_{x}=0, \\
& v_{t}+g(u, v)_{x}=0
\end{aligned}
$$

with arbitrary initial constant states

$$
\left(u_{0}(x), v_{0}(x)\right)= \begin{cases}\left(u_{l}, v_{l}\right), & x<0, \\ \left(u_{r}, v_{r}\right), & x>0,\end{cases}
$$

is considered. We assume that $f_{v}<0, g_{u}<0$. Let $l_{i}\left(r_{i}\right)$ be the left (right) eigenvectors of $d F \equiv d(f, g)$ for eigenvalues $\lambda_{1}<\lambda_{2}$. Instead of assuming the usual convexity condition $d \lambda_{i}\left(r_{i}\right) \neq 0, i=1,2$, we assume that $d \lambda_{i}\left(r_{i}\right)=0$ on disjoint union of 1-dim manifolds in the $(u, v)$ plane. Oleinik's condition (E) for single equation is extended to system (1); again call this new condition (E). Our condition (E) implies Lax's shock inequalities and, in case $d \lambda_{i}\left(r_{i}\right) \neq 0$, the two are equivalent. We then prove that there exists a unique solution to the Riemann Problem (1) and (2) in the class of shocks, rarefaction waves and contact discontinuities which satisfies condition (E).

Introduction. We consider the system

$$
\begin{aligned}
& u_{t}+f(u, v)_{x}=0, \\
& v_{t}+g(u, v)_{x}=0, \quad t \geqslant 0,-\infty<x<\infty,
\end{aligned}
$$

where $u=u(x, t), v=v(x, t)$ and $f, g \in C^{3}(U)$ for some open set $U$ in $R^{2}$.

The problem to be solved is the Riemann Problem $\left\{\left(u_{l}, v_{l}\right) ;\left(u_{r}, v_{r}\right)\right\}$ for arbitrary constants $\left(u_{l} v_{l}\right) \in U,\left(u_{r}, v_{r}\right) \in U$; i.e. solve the system $(0.1)$ with initial data

$$
(u(x, 0), v(x, 0)) \equiv\left(u_{0}(x), v_{0}(x)\right)= \begin{cases}u_{l}, v_{l} & \text { for } x<0 \\ u_{r}, v_{r} & \text { for } x>0\end{cases}
$$

Presented to the Society, January 26, 1972; received by the editors October 16, 1973.

AMS (MOS) subject classifications (1970). Primary 35L65, 35F25.

Key words and phrases. Conservation laws, shocks $S$, rarefaction waves $R$, contact discontinuities, Oleinik condition (E), Lax shock inequalities (L), shock speed.

(1) In part this paper represents the research included in a doctoral dissertation submitted by the author at the University of Michigan in 1973. 
Besides the physical meaning, the significance of the Riemann Problem is that it is commonly served to solve the Cauchy Problem (0.1) with general initial data. In fact, Riemann Problems are the building blocks in the proof of existence theorems in Glimm [1], Smoller and Johnson [2], Nishida [4] and Nishida and Smoller [5].

Since the solution to $(0.1)$ is usually discontinuous, see e.g. [3], we make the following definition.

DEFINITION. The bounded measurable function $(u, v)$ is said to be a weak solution to $(0.1),(0.2)$ if

$$
\begin{aligned}
& \iint_{t \geqslant 0}\left[u \phi_{t}+f(u, v) \phi_{x}\right] d x d t+\int_{t=0} u_{0} \phi d x=0, \\
& \iint_{t \geqslant 0}\left[v \phi_{t}+g(u, v) \phi_{x}\right] d x d t+\int_{t=0} v_{0} \phi d x=0
\end{aligned}
$$

for all smooth functions $\phi=\phi(t, x)$ with compact support in $t \geqslant 0$.

Hereafter, we assume that, for $(u, v) \in U$,

$$
\begin{array}{ll}
f_{v}<0, & g_{u}<0, \\
f_{u} \geqslant 0, & g_{v} \leqslant 0 .
\end{array}
$$

Let $F=(f, g), d F$ the Fréchet derivative of $F$ and $d^{2} F$ the Fréchet derivative of $d F$. Condition (0.4) implies that system (0.1) is hyperbolic, i.e. $d F$ has real and distinct eigenvalues $\lambda_{1}<\lambda_{2} .(0.5)$ implies that, for $(u, v) \in U$,

$$
\lambda_{1}<0<\lambda_{2} \text {. }
$$

Let $r_{i}$ (resp. $l_{i}$ ) be right (resp. left) eigenvectors corresponding to eigenvalues $\lambda_{i}, i=1,2$. These can be taken in the form

$$
\begin{array}{ll}
r_{1}=\left(1, a_{1}\right)^{t}, & r_{2}=\left(1, a_{2}\right)^{t}, \\
l_{1}=\left(-a_{2}, 1\right), & l_{2}=\left(-a_{1}, 1\right),
\end{array}
$$

where

$$
\begin{aligned}
& a_{i}=\frac{g_{u}}{\lambda_{i}-g_{v}}=\frac{\lambda_{i}-f_{u}}{f_{v}}, \quad i=1,2, \\
& a_{2}<0<a_{1} .
\end{aligned}
$$

If $d \lambda_{i} \cdot r_{i} \neq 0$, then system (0.1) is said to be genuinely nonlinear in the $i$ th class.

Suppose $(u, v)$ is a solution which is discontinuous across curve $x=x(t)$, then (0.3) implies the following Hugoniot condition (e.g. [3]) 


$$
s=\frac{f\left(u_{1}, v_{1}\right)-f\left(u_{0}, v_{0}\right)}{u_{1}-u_{0}}=\frac{g\left(u_{1}, v_{1}\right)-g\left(u_{0}, v_{0}\right)}{v_{1}-v_{0}} \equiv \sigma\left(u_{0}, v_{0} ; u_{1}, v_{1}\right)
$$

where $s=\dot{x}(t),\left(u_{0}, v_{0}\right)=(u, v)(x-0, t),\left(u_{1}, v_{1}\right)=(u, v)(x+0, t)$.

Definition. For $\left(u_{0}, v_{0}\right) \in U$, the shock curve $s\left(u_{0}, v_{0}\right)$ is the set $(u, v) \in U$ which satisfies the Hugoniot condition

$$
\frac{f(u, v)-f\left(u_{0}, v_{0}\right)}{u-u_{0}}=\frac{g(u, v)-g\left(u_{0}, v_{0}\right)}{v-v_{0}}
$$

The forward shock curve $S_{2}\left(u_{0}, v_{0}\right)$ and backward shock curve $S_{1}\left(u_{0}, v_{0}\right)$ are:

$$
\begin{aligned}
& S_{1}\left(u_{0}, v_{0}\right)=S\left(u_{0}, v_{0}\right) \cap\left\{(u, v) \mid u \geqslant u_{0}, v \geqslant v_{0} \text { or } u \leqslant u_{0}, v \leqslant v_{0}\right\}, \\
& S_{2}\left(u_{0}, v_{0}\right)=S\left(u_{0}, v_{0}\right) \cap\left\{(u, v) \mid u \geqslant u_{0}, v \leqslant v_{0} \text { or } u \leqslant u_{0}, v \geqslant v_{0}\right\} .
\end{aligned}
$$

Let $\left(u_{1}, v_{1}\right) \in S_{2}\left(u_{0}, v_{0}\right)$, then we can define a weak solution $(u, v)$ to (0.1) by

$$
i(u, v)(x, t)= \begin{cases}\left(u_{0}, v_{0}\right) & \text { for } x<\sigma t \\ \left(v_{1}, v_{1}\right) & \text { for } x>\sigma t\end{cases}
$$

where

$$
\sigma=\sigma\left(u_{1}, v_{1} ; u_{0}, v_{0}\right)=\frac{f\left(u_{1}, v_{1}\right)-f\left(u_{0}, v_{0}\right)}{u_{1}-u_{0}}=\frac{g\left(u_{1}, u_{1}\right)-g\left(u_{0}, v_{0}\right)}{v_{1}-v_{0}}
$$

We call such solution a forward shock wave. Similarly, for a $S_{2}$ curve, we have backward shock wave. We denote them by the following pictures.

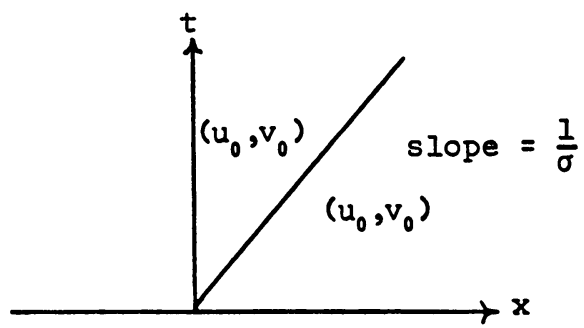

forward shock wave

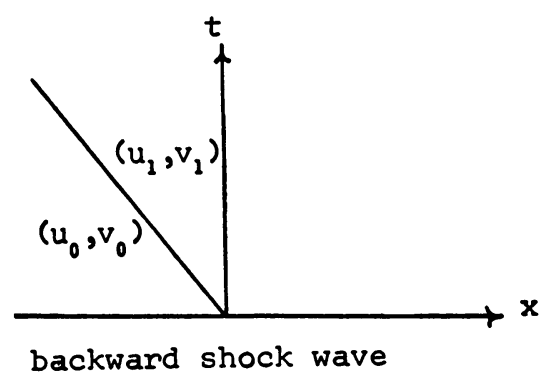

backward shock wave

Let

$$
\begin{aligned}
& V_{ \pm}^{2}=\left\{(u, v) \in U \mid d \lambda_{2}\left(r_{2}\right) \gtrless 0\right\}, \\
& V_{ \pm}^{1}=\left\{(u, v) \in U \mid d \lambda_{1}\left(r_{1}\right) \gtrless 0\right\} .
\end{aligned}
$$

Let $\left(u_{1}, v_{1}\right) \in R_{2}\left(u_{0}, v_{0}\right), u_{1}>u_{0}$, be such that every point $(u, v)$ on $R_{2}\left(u_{0}, v_{0}\right)$ between $\left(u_{0}, v_{0}\right)$ and $\left(u_{1}, v_{1}\right)$ lies in region $V_{-}^{2}$. Then the Riemann Problem $\left\{\left(u_{0}, v_{0}\right) ;\left(u_{1}, v_{1}\right)\right\}$ can be solved by (cf. [3]) 


$$
(u, v)(x, t)=(\tilde{u}, \tilde{v})(x / t)
$$

$$
\lambda_{2}(\tilde{u}, \tilde{v})(x / t)=x / t
$$

$$
(\tilde{u}, \tilde{v}) \in R_{2}\left(u_{0}, v_{0}\right), u_{1} \geqslant \tilde{u} \geqslant u_{0}
$$

We call this solution, which takes values along a rarefaction curve $R_{2}$, a forward rarefaction wave. We can also have a forward rarefaction wave when $u_{1}<u_{0}$ and the corresponding region is $V_{+}^{2}$. The backward rarefaction wave, which takes values on a $R_{1}$ curve can be treated similarly. These can be pictured as:
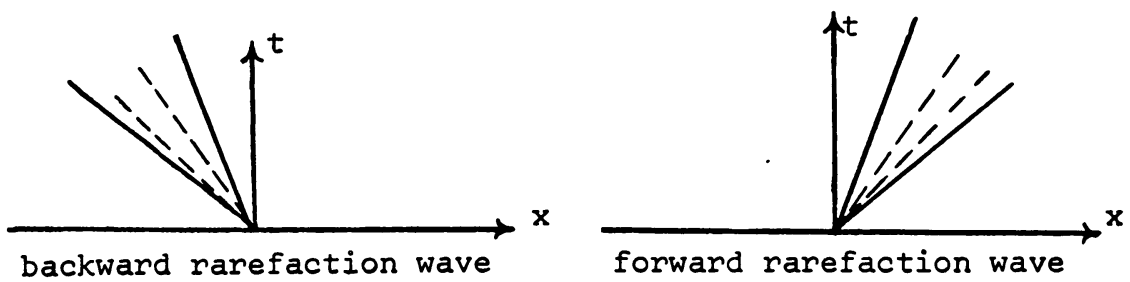

Joel A. Smoller [7] and [8] solved the Riemann Problem in the class of shock and rarefaction waves under the assumption that system $(0.1)$ is genuinely nonlinear and that the shock interaction condition holds. The solution is required to satisfy the following Lax shock inequalities, e.g. [3], across shocks:

(L) or

$$
\lambda_{1}\left(u_{0}, v_{0}\right)>\sigma>\lambda_{1}\left(u_{1}, v_{1}\right) \text { and } \sigma<\lambda_{2}\left(u_{1}, v_{1}\right)
$$

$$
\lambda_{2}\left(u_{0}, v_{0}\right)>\sigma>\lambda_{2}\left(u_{1}, v_{1}\right) \text { and } \sigma>\lambda_{1}\left(u_{0}, v_{0}\right)
$$

where $\sigma=\sigma\left(u_{0}, v_{0} ; u_{1}, v_{1}\right)$.

The purpose of this paper is to prove the existence and uniqueness theorem for the Riemann Problem in the class of shocks, rarefaction waves and contact discontinuities, when we relax the genuine-nonlinearity condition and let $d \lambda_{i}\left(r_{i}\right)=$ $0, i=1,2$, on a disjoint union of 1 -manifolds in the $(u, v)$ plane. The solution is required to satisfy, instead of condition $(L)$, the following extended entropy condition

$$
\sigma\left(u_{1}, v_{1} ; u_{0}, v_{0}\right) \leqslant \sigma\left(u, v ; u_{0}, v_{0}\right)
$$

for every $(u, v)$ on $S\left(u_{0} ; v_{0}\right)$ between $\left(u_{0}, v_{0}\right)$ and $\left(u_{1}, v_{1}\right)$.

Condition (E) extends Oleinik's celebrated condition (E) (cf. [6]) to systems and reduces to condition $(\mathrm{L})$ when the system $(0.1)$ is genuinely nonlinear.

1. Preliminary results. In this section, we shall make some basic observations about the shock and rarefaction curves.

Let $d / d \mu$ (resp. $d / d \nu_{i}$ ) be the derivative along curve $S_{2}$ (resp. $R_{i}$ ). Thus, 


$$
\begin{aligned}
\frac{d}{d \mu} & =\frac{d}{d u}+h_{2} \frac{d}{d v}, \\
h_{2}=h_{2}\left(u_{0}, v_{0} ; u, v\right) & =\frac{\left(u-u_{0}\right) g_{u}+\left(\sigma-f_{u}\right)\left(v-v_{0}\right)}{\left(v-v_{0}\right) f_{v}+\left(\sigma-g_{v}\right)\left(u-u_{0}\right)} \\
\sigma & =\sigma\left(u_{0}, v_{0} ; u, v\right)=\frac{f(u, v)-f\left(u_{0}, v_{0}\right)}{u-u_{0}}=\frac{g(u, v)-g\left(u_{0}, v_{0}\right)}{v-v_{0}} \\
\frac{d}{d v_{i}} & =\frac{d}{d u}+a_{i} \frac{d}{d v}, \quad i=1,2
\end{aligned}
$$

where $g_{u}, g_{v}, f_{u}, f_{v}$ and $a_{i}, i=1,2$, are evaluated at $(u, v)$.

LEMMA 1.1. For $\left(u_{1}, v_{1}\right) \in S_{2}\left(u_{0}, v_{0}\right)$ (resp. $\left.S_{1}\left(u_{0}, v_{0}\right)\right)$, we have $\sigma\left(u_{1}, v_{1} ; u_{0}, v_{0}\right) \geqslant 0$ (resp. $\left.\leqslant 0\right)$.

LEMMA 1.2. The set $S_{2}\left(u_{0}, v_{0}\right)$ (resp. $S_{1}\left(u_{0}, v_{0}\right)$ ) is a smooth curve defined for all $u$ (resp. $v)$.

Lemmas 1.1 and 1.2 can be proved by using (0.4) and (0.5); we omit the propofs.

LEMMA 1.3. Given $\left(u_{0}, v_{0}\right) \in U$, and supposing that for any $(u, v)$ on $S_{2}\left(u_{0}, v_{0}\right), u>u_{0},\left|u-u_{0}\right|$ small, we have $(u, v) \in V_{+}^{2}$. Then, for such $(u, v)$,

$$
\begin{aligned}
\lambda_{2}(u, v) & <\sigma\left(u, v ; u_{0}, v_{0}\right)<\lambda_{2}\left(u_{0}, v_{0}\right) \\
\sigma\left(u, v ; u_{0}, v_{0}\right) & =\lambda_{2}\left(u_{0}, v_{0}\right)+O\left|u-u_{0}\right| ; \\
h_{2}\left(u, v ; u_{0}, v_{0}\right) & =a_{2}\left(u_{0}, v_{0}\right)+O\left|u-u_{0}\right|
\end{aligned}
$$

and $\sigma$ is decreasing along $S_{2}\left(u_{0}, v_{0}\right)$.

Proof. See Lax [3].

LEMMA 1.4. For any $(u, v) \in S_{2}\left(u_{0}, v_{0}\right), \sigma=\sigma\left(u_{0}, v_{0} ; u, v\right), u>u_{0}$, the following are equivalent:

(i) $d \sigma / d \mu>0$ (resp. $d \sigma / d \mu<0$ ).

(ii) $\sigma<\lambda_{2}$ (resp. $\sigma>\lambda_{2}$ ).

If $u<u_{0}$, then the following are equivalent:

(iii) $d \sigma / d \mu>0$ (resp. $d \sigma / d \mu<0$ ).

(iv) $\sigma>\lambda_{2}$ (resp. $\sigma<\lambda_{2}$ ).

Thus $d \sigma / d \mu=0$ if and only if $\sigma=\lambda_{2}$.

Proof. We only consider the case $u>u_{0}$. Thus, by Lemma 1.2, $v<v_{0}$.

Case 1: $h_{2} \geqslant a_{2}$ at $(u, v)$. Assume (i), $f_{u}+h_{2} f_{v}-\sigma>0$; thus $\lambda_{2}=$ $f_{u}+a_{2} f_{v} \geqslant f_{u}+h_{2} f_{v}>\sigma$ which is (ii). 
Assume (ii), then

$$
\frac{d \sigma}{d \mu}=\frac{g_{u}+h_{2} g_{v}-\sigma h_{2}}{v-v_{0}} \geqslant \frac{g_{u}+a_{2} g_{v}-\sigma a_{2}}{v-v_{0}}>\frac{g_{u}+a_{2} g_{v}-\lambda_{2} a_{2}}{v-v_{0}}=0
$$

which is (i).

Case 2: $h_{2}<a_{2}$ at $(u, v)$. Assume (i), $g_{u}+h_{2} g_{v}-\sigma h_{2}<0$, thus

$$
0<g_{u}+h_{2} g_{v}-\sigma h_{2}<g_{u}+a_{2} g_{v}-a_{2} \sigma=a_{2} \lambda_{2}-a_{2} \sigma=a_{2}\left(\lambda_{2}-\sigma\right) \text {. }
$$

So $\lambda_{2}-\sigma>0$ which is (ii).

Assume (ii), then

$$
\begin{aligned}
\frac{d \sigma}{d \mu} & =\frac{f_{u}+h_{2} f_{v}-\sigma}{u-u_{0}}>\frac{f_{u}+h_{2} f_{v}-\lambda_{2}}{u-u_{0}}=\frac{f_{u}+h_{2} f_{v}-\left(f_{u}+a_{2} f_{v}\right)}{u-u_{0}} \\
& =\frac{\left(h_{2}-a_{2}\right) f_{v}}{u-u_{0}}>0
\end{aligned}
$$

which is (i).

The fact that $d \sigma / d \mu<0$ and $\sigma>\lambda_{2}$ are equivalent can be proved similarly. Q.E.D.

Using Lemmas 1.3 and 1.4 we can easily prove the following:

LEMMA 1.5. If $\sigma=\lambda_{2}$ at $(u, v) \in S_{2}\left(u_{0}, v_{0}\right)$, then $h_{2}=a_{2}$ at $(u, v)$.

LEMMA 1.6. Let $(u, v) S_{2}\left(u_{0}, v_{0}\right) \in V_{+}^{2}$ and $\lambda_{2}=\sigma$ at $(u, v)$. Then $d\left(\sigma-\lambda_{2}\right) / d \mu=d \lambda_{2} / d \mu>0$ and $\sigma$ has local maximum at $(u, v)$.

LEMMA 1.7. Suppose $\left(u_{1}, v_{1}\right) \in S_{2}\left(u_{0}, v_{0}\right)$ and condition (E) is satisfied for $\left\{\left(u_{0}, v_{0}\right) ;\left(u_{1}, v_{1}\right)\right\}$. Then, for $\sigma=\sigma\left(u_{0}, v_{0} ; u_{1}, v_{1}\right)$,

$$
\lambda_{1}\left(u_{0}, v_{0}\right) \leqslant \sigma \text { and } \lambda_{2}\left(u_{0}, v_{0}\right) \geqslant \sigma \geqslant \lambda_{2}\left(u_{1}, v_{1}\right)
$$

We have analogous lemmas as above for $S_{1}\left(u_{0}, v_{0}\right)$; in this case, we use $d / d \mu=k d / d u+d / d v$,

$$
k=k\left(u_{0}, v_{0} ; u, v\right)=\frac{\left(\sigma-g_{v}\right)\left(u-u_{0}\right)+f_{v}\left(v-v_{0}\right)}{\left(\sigma-f_{u}\right)\left(v-v_{0}\right)+g_{v}\left(u-u_{0}\right)}
$$

THEOREM 1.1. Condition (E) is equivalent to Lax's shock inequalities when system (0.1) is genuinely nonlinear. 
Proof. We only prove the necessity part of the theorem for forward shocks. The other cases can be treated similarly. Thus we assume that $\left(u_{1}, v_{1}\right) \in$ $S_{2}\left(u_{0}, v_{0}\right)$ and $\left\{\left(u_{0}, v_{0}\right) ;\left(u_{1}, v_{1}\right)\right\}$ satisfies condition (E). Without loss of generality, assume $d \lambda_{2}\left(r_{2}\right)<0$.

If $u_{1}>u_{0}$, then, by Lemma $1.3, \lambda_{2}<\sigma$ at all points on $S_{2}\left(u_{0}, v_{0}\right)$ between $\left(u_{0}, v_{0}\right)$ and $\left(u_{0}, v_{0}\right)$ and close to $\left(u_{0}, v_{0}\right)$. For such points, by Lemma 1.4, we have $d \sigma / d \mu<0$. We claim that, for all points on $S_{2}\left(u_{0}, v_{0}\right)$ between $\left(u_{0}, v_{0}\right)$ and $\left(u_{1}, v_{1}\right)$, we actually have $d \sigma / d \mu<0$. Indeed if $(u, v)$ is the first point on $S_{2}\left(u_{0}, v_{0}\right), u>u_{0}$, such that $d \sigma / d \mu=0$, then, by Lemma 1.6, $\sigma<\lambda_{2}$ at some point $(\widetilde{u}, \widetilde{v})$ on $S_{2}\left(u_{0}, v_{0}\right)$ between $\left(u_{0}, v_{0}\right)$ and $(u, v)$. Thus, by Lemma 1.4, $d \sigma / d \mu>0$ at $(\tilde{u}, \widetilde{v})$. But since $d \sigma / d \mu<0$ at points close to $\left(u_{0}, v_{0}\right)$, we then have $d \sigma / d \mu=0$ at some point between $\left(u_{0}, v_{0}\right)$ and $(\tilde{u}, \widetilde{v})$. This contradicts the fact that $(u, v)$ is the first point with $d \sigma / d \mu=0$. So we have $d \sigma / d \mu<0$ at all points on $S_{2}\left(u_{0}, v_{0}\right)$ between $\left(u_{0}, v_{0}\right)$ and $\left(u_{1}, v_{1}\right)$. In particular, $\sigma\left(u_{1}, v_{1} ; u_{0}, v_{0}\right)<\sigma\left(u_{0}, v_{0} ; u_{0}, v_{0}\right)=\lambda_{2}\left(u_{0}, v_{0}\right)$ and $\sigma\left(u_{1}, v_{1} ; u_{0}, v_{0}\right)>\lambda_{2}\left(u_{1}, v_{1}\right)$ by Lemma 1.4. Since $\sigma>0, \lambda_{1}<0<\lambda_{2}$, we have condition (L).

If $u_{1}<u_{0}$, a similar argument gives that $\sigma$ is increasing as $(u, v)$ moves from $\left(u_{0}, v_{0}\right)$ to $\left(u_{1}, v_{1}\right)$ along $S_{2}\left(u_{0}, v_{0}\right)$. This would contradict condition (E). Hence $u_{1}>u_{0}$ and we are done. Q.E.D.

2. Existence. In this section, we want to solve the Riemann Problem in the class of shocks, rarefaction waves and contact discontinuities.

We make the following assumption:

If $d \lambda_{i}\left(r_{i}\right)=0$ at point $(u, v)$, then $d\left(d \lambda_{i}\left(r_{i}\right)\right) / d v_{i} \neq 0, i=1,2$, where $d / d v_{i}=d / d u+a_{i} d / d v$.

LEMmA 2.1. The set $V_{0}^{i}=\left\{(u, v) \mid d \lambda_{i}\left(r_{i}\right)=0\right\}$ is union of disjoint 1-manifolds and transversal to integral curves of $d v / d u=a_{i}, i=1,2$.

LEMMA 2.2. Given $(u, v) \in S_{2}\left(u_{0}, v_{0}\right) \cap V_{0}^{2}, \sigma=\sigma\left(u_{0}, v_{0} ; u, v\right)=$ $\lambda_{2}(u, v)=\lambda_{2}, u>u_{0}$, we have, at $(u, v)$,

$$
\frac{d \sigma}{d \mu}=\frac{d \lambda_{2}}{d \mu}=\frac{d^{2} \sigma}{d \mu^{2}}=0 \text { and } \frac{d^{2} \lambda_{2}}{d \mu^{2}}>0
$$

and $\sigma$ is increasing at $(u, v)$ if immediately to the right of $(u, v)$ along $R_{2}(u, v)$ is region $V_{-}^{2}, d^{2} \lambda_{2} / d \mu^{2}<0$ and $\sigma$ is decreasing at $(u, v)$ if immediately to the left of $(u, v)$ along $R_{2}(u, v)$ is region $V_{+}^{2}$. 
Lemmas 2.1 and 2.2 are easy consequences of (2.1) and Lemma 1.5; we omit the proofs.

Before we state and prove our rather long existence theorem, we sketch the construction of the solution.

Given a fixed point $\left(u_{0}, v_{0}\right)$ in $U$, we first construct a curve $\gamma\left(u_{0}, v_{0}\right)=\gamma$ so that points $(u, v)$ on $\gamma, u>u_{0}, v<v_{0}$, can be connected to $\left(u_{0}, v_{0}\right)$ on the right by forward waves.

Suppose $\left(u_{0}, v_{0}\right) \in V_{+}^{2}$; we then let the first segment of $\gamma$ be $S_{2}\left(u_{0}, v_{0}\right)$ and so the solution can be pictured as

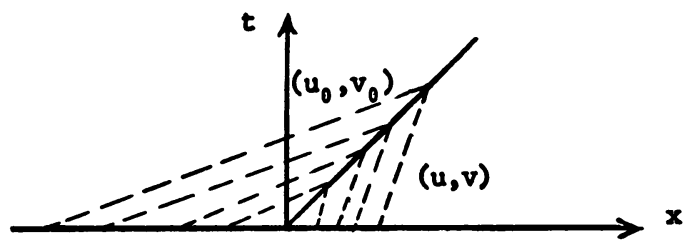

which is a forward shock, (where light lines denote characteristic lines).

As $(u, v)$ moves further to the right along $S_{2}\left(u_{0}, v_{0}\right)$, the picture becomes at some point $\left(u_{1}, v_{1}\right)$,

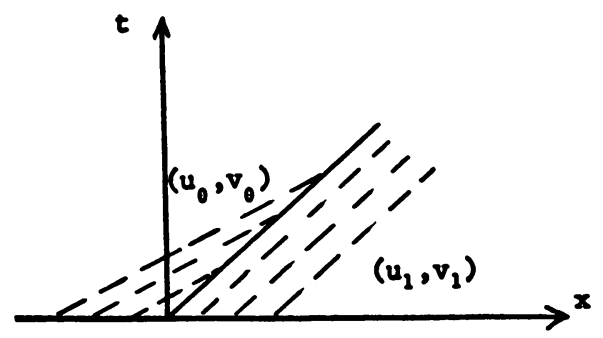

i.e. $\sigma\left(u_{0}, v_{0} ; u_{1}, v_{1}\right)=\lambda_{2}\left(u_{1}, v_{1}\right)$. We then continue $\gamma$ by $R_{2}\left(u_{1}, v_{1}\right)$, so that the solution is a shock connecting $\left(u_{0}, v_{0}\right)$ and $\left(u_{1}, v_{1}\right)$ followed by a rarefaction wave connecting $\left(u_{1}, v_{1}\right)$ and $(u, v)$ on $R_{2}\left(u_{1}, v_{1}\right)$. The diagram is

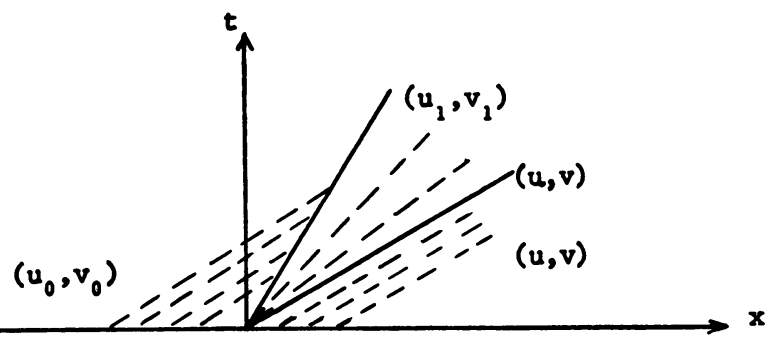

When $R_{2}\left(u_{1}, v_{1}\right)$ leaves region $V_{-}^{2}$ at $\left(u_{2}, v_{2}\right)$, we continue $\gamma$ so that 
the point $(u, v)$ can be connected by shock to some $\left(u^{*}, v^{*}\right)$ on $R_{2}\left(u_{1}, v_{1}\right)$ between $\left(u_{1}, v_{1}\right)$ and $\left(u_{2}, v_{2}\right)$ and $\lambda_{2}\left(u^{*}, v^{*}\right)=\sigma\left(u^{*}, v^{*} ; u, v\right)$. This can be pictured as

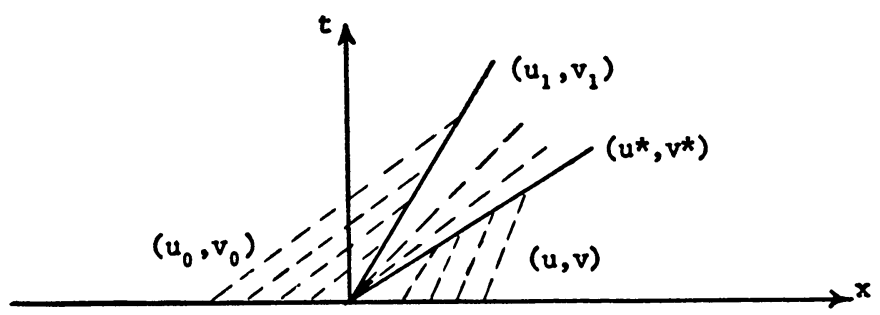

Continue $\gamma$ until some point $\left(u_{3}, v_{3}\right)$ so that $\sigma\left(u_{3}^{*}, v_{3}^{*} ; u_{3}, v_{3}\right)=$ $\lambda_{2}\left(u_{3}, v_{3}\right)$. We then continue $\gamma$ by $R_{2}\left(u_{3}, v_{3}\right)$ and point $(u, v)$ on $R_{2}\left(u_{3}, v_{3}\right)$ is connected to $\left(u_{3}, v_{3}\right)$ by rarefaction wave:

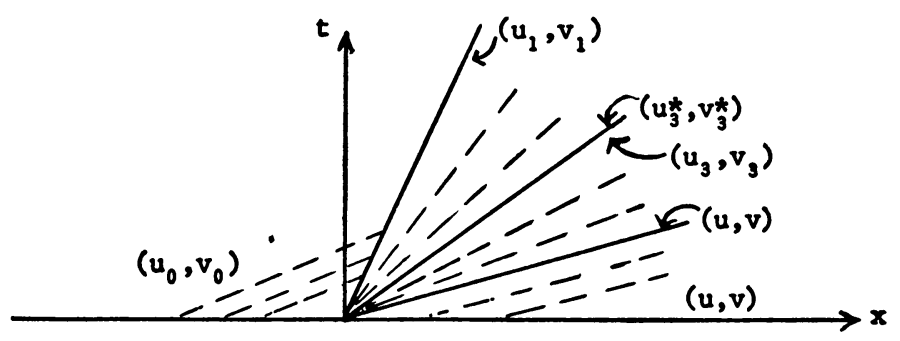

Continue these processes so that $\gamma$ is defined for all $u>u_{0}$. During the process we must always make sure that condition (E) holds for any discontinuity.

To be precise, we construct the curve $\gamma$ as follows:

Step 1. By Lemma 2.1, we know that any curve in $V_{0}^{2}$ is transversal to $R_{2}$ curves, so we have two cases:

(i) Immediately to the right of $\left(u_{0}, v_{0}\right)$ along $R_{2}\left(u_{0}, v_{0}\right)$ is region $V_{+}^{2}$ :

The curve $\gamma_{1}$, the first segment of $\gamma$, is $S_{2}\left(u_{0}, v_{0}\right)$ starting at $\left(u_{0}, v_{0}\right)$ and extended until there exists a point $\left(u_{1}, v_{1}\right)$ which is the first point on $S_{2}\left(u_{0}, v_{0}\right)$ such that $\lambda_{2}\left(u_{1}, v_{1}\right)=\sigma\left(u_{0}, v_{0} ; u_{1}, v_{1}\right)$ and immediately to the right of $\left(u_{1}, v_{1}\right)$ along $R_{2}\left(u_{1}, v_{1}\right)$ is region $V_{-}^{2}$. The point $(u, v)$ on $\gamma_{1}$ is connected to $\left(u_{0}, v_{0}\right)$ by a forward shock. Analogous to the proof of Theorem 1.1, with the aid of Lemma 2.2, we can show that $\sigma$ is decreasing between $\left(u_{0}, v_{0}\right)$ and $\left(u_{1}, v_{1}\right)$. Thus condition $(\mathrm{E})$ is clearly satisfied for $\left\{\left(u_{0}, v_{0}\right)\right.$, $(u, v)\},(u, v) \in \gamma_{1}$.

(ii) Immediately to the right of $\left(u_{0}, v_{0}\right)$ along $R_{2}\left(u_{0}, v_{0}\right)$ is region $V_{-}^{2}$ :

The curve $\gamma_{1}$ is $R_{2}\left(u_{0}, v_{0}\right)$ starting at $\left(u_{0}, v_{0}\right)$ and extended to $\left(u_{1}, v_{1}\right)$, the first point at which $R_{2}\left(u_{0}, v_{0}\right)$ leaves $V_{-}^{2}$. Point $(u, v)$ on $\gamma_{1}$ is connected to $\left(u_{0}, v_{0}\right)$ by a forward rarefaction wave. 
Step 2. (i) For case (i) of Step 1:

Since immediately to the right of $\left(u_{1}, v_{1}\right)$ is region $V_{-2}^{2}$, we proceed as in case (ii) of Step 1, i.e. continue the curve from $\left(u_{1}, v_{1}\right)$ by $R_{2}\left(u_{1}, v_{1}\right)$ until there exists a point $\left(u_{2}, v_{2}\right)$ at which $R_{2}\left(u_{1}, v_{1}\right)$ first leaves region $V_{-}^{2}$. The point $(u, v)$ on $R_{2}\left(u_{1}, v_{1}\right)$ between $\left(u_{1}, v_{1}\right)$ and $\left(u_{2}, v_{2}\right)$ is connected to $\left(u_{1}, v_{1}\right)$ by a forward rarefaction wave. The diagrams look like:

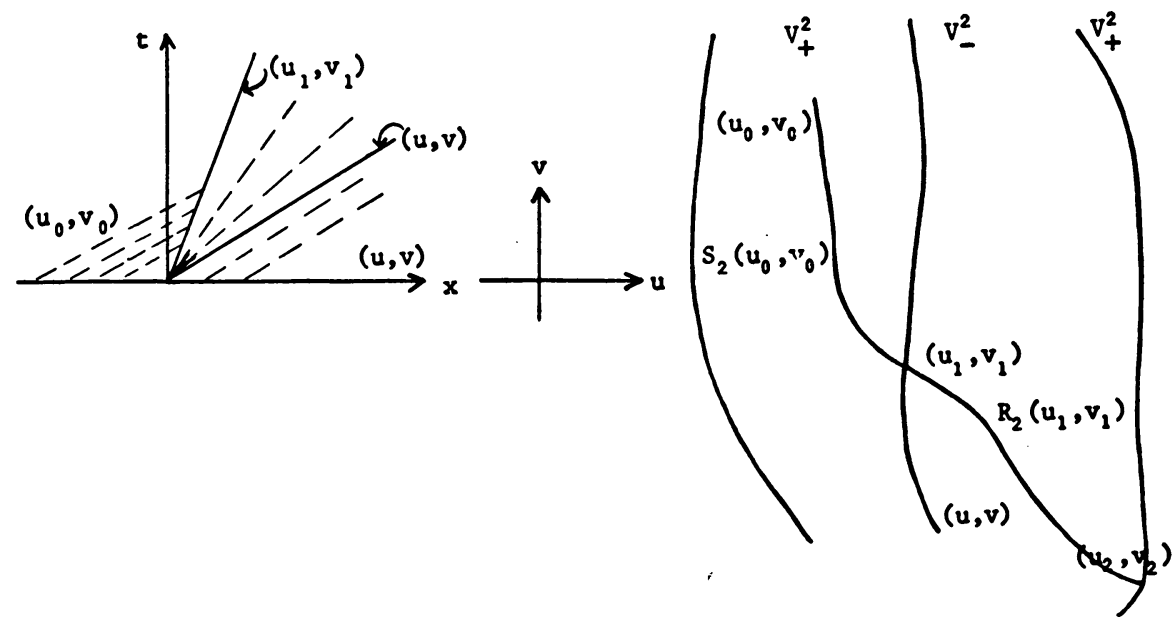

(ii) For case (ii) of Step 1:

We continue the curve from $\left(u_{1}, v_{1}\right)$ by a mixed curve $\gamma_{1}^{*}$ defined as follows: $(u, v) \in \gamma_{1}^{*}$ if there is a point $\left(u^{*}, v^{*}\right) \in \gamma_{1}$ such that $(u, v)$ is the first point on $S_{2}\left(u^{*}, v^{*}\right), u>u^{*}$, at which $\sigma\left(u^{*}, v^{*}, u, v\right)=\lambda_{2}\left(u^{*}, v^{*}\right)$. Such a $\gamma_{1}^{*}$ exists at least when $\left|u^{*}-u_{1}\right|$ is small. In fact, since immediately to the right of $\left(u_{1}, v_{1}\right)$ is region $V_{+}^{2}$, we know that $\sigma\left(u_{1}, v_{1} ; u, v\right)$ is decreasing. By continuity of $\sigma$, we then have, for $\left(\widetilde{u}^{*}, \widetilde{v}^{*}\right) \in \gamma_{1}$ and near $\left(u_{1}, v_{1}\right)$, the following diagram:

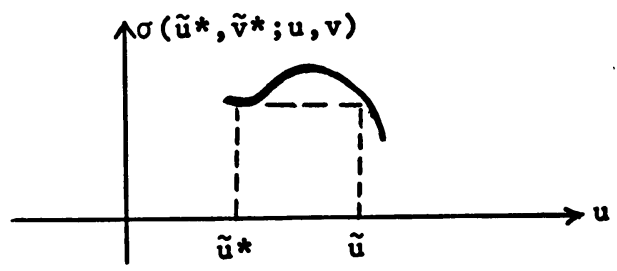

The reason $d \sigma / d \mu>0$ near $\left(\widetilde{u}^{*}, \widetilde{v}^{*}\right)$ is that $\left(\widetilde{u}^{*}, \widetilde{v}^{*}\right)$ lies in region $V_{-}^{2}$.

We then connect $\left(u^{*}, v^{*}\right)$ on $\gamma_{1}$ to $(u, v)$ on $\gamma_{1}^{*}$ by shock wave. Since $\left(u^{*}, v^{*}\right) \in \gamma_{1} \subset V_{-}^{2}$, by definition of $\gamma_{1}^{*}$, condition (E) is satisfied for $\left\{\left(u^{*}, v^{*}\right) ;(u, v)\right\}$. Indeed, the diagrams look like the following: 

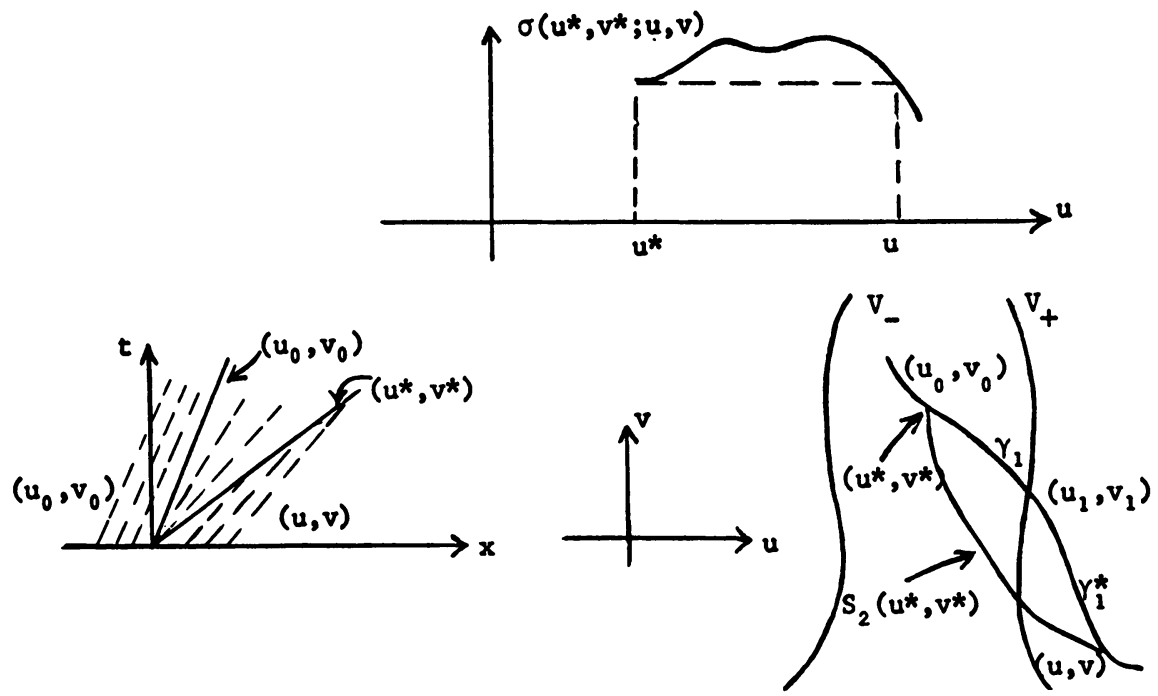

We continue the curve by $\gamma_{1}^{*}$ starting at $\left(u_{1}, v_{1}\right)$ until point $\left(u_{2}, v_{2}\right)$ defined as follows:

(ii) (a) There exists point $\left(u_{2}, v_{2}\right)$ on $\gamma_{1}^{*}$ such that the corresponding point $\left(u_{2}^{*}, v_{2}^{*}\right)=\left(u_{0}, v_{0}\right)$. In this case we continue the curve from $\left(u_{2}, v_{2}\right)$ by $S_{2}\left(u_{0}, v_{0}\right)$ and point $(u, v)$ on $S_{2}\left(u_{0}, v_{0}\right)$ is connected to $\left(u_{0}, v_{0}\right)$ by a forward shock. Condition (E) is clearly satisfied for those $(u, v) \in S_{2}\left(u_{0}, v_{0}\right)$ and close to $\left(u_{2}, v_{2}\right)$. We have diagrams like the following:
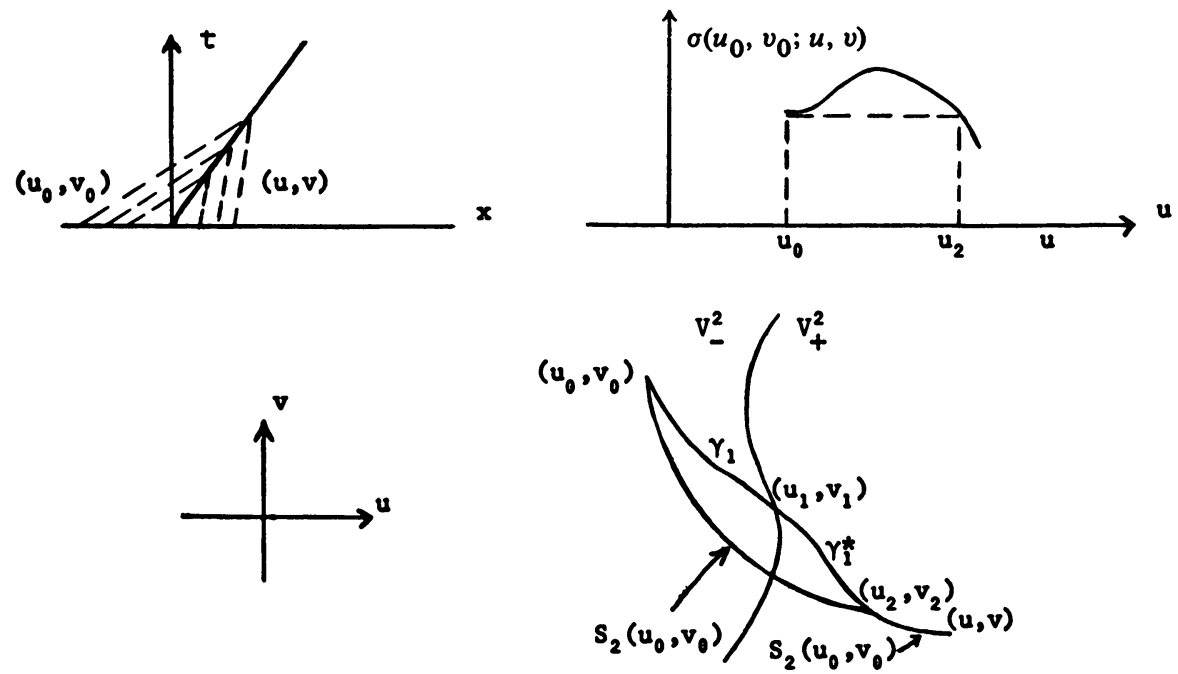

(ii) (b) There exists a point $\left(u_{2}, v_{2}\right)$ on $\gamma_{1}^{*}$ and a corresponding point $\left(u_{2}^{*}, v_{2}^{*}\right)$ on $\gamma_{1}$ such that $\sigma\left(u_{2}^{*}, v_{2}^{*}, u, v\right)$ attains local minimum at $\left(u_{2}, v_{2}\right)$. 
For this case, we know, by Lemmas $1.4,1.6$ and 2.1 that $\sigma\left(u_{2}^{*}, v_{2}^{*} ; u_{2}, v_{2}\right)=$ $\lambda_{2}\left(u_{2}, v_{2}\right)$ and $\left(u_{2}, v_{2}\right) \in V_{-}^{2}$. We then continue the curve from $\left(u_{2}, v_{2}\right)$ by $R_{2}\left(u_{2}, v_{2}\right)$ and the point $(u, v) \in R_{2}\left(u_{2}, v_{2}\right)$ is connected to $\left(u_{2}, v_{2}\right)$ by a forward wave. The diagrams look like the following:
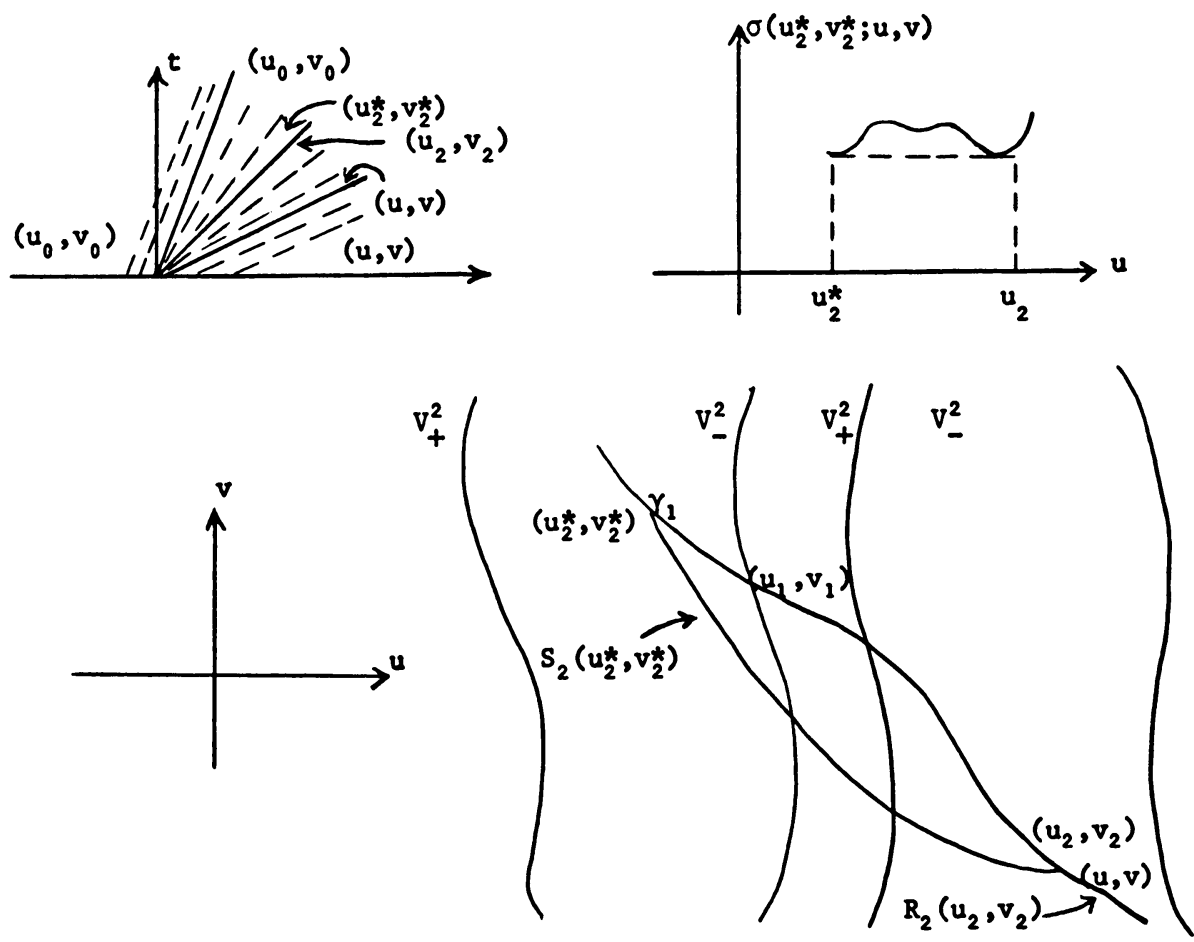

The discontinuous part of the solution $\left(u_{2}^{*}, v_{2}^{*}\right),\left(u_{2}, v_{2}\right)$ has the property that the shock speed $\sigma$ coincides with eigenvalue $\lambda_{2}$ on either side. We call such discontinuity a contact discontinuity.

REMARK. Lemma 2.3-Lemma 2.5 to be presented later will show that a mixed curve is continuous and decreasing as a function of $u$.

Step 3. (i) For case (i) of Step 2:

We continue the curve by the mixed curve $\gamma_{2}^{*}$ starting at $\left(u_{2}, v_{2}\right)$ where $\gamma_{2}$ is the rarefaction curve $R_{2}\left(u_{1}, v_{1}\right)$ between $\left(u_{1}, v_{1}\right)$ and $\left(u_{2}, v_{2}\right)$. The process is exactly the same as in case (ii) of Step 2 .

If there is a point $\left(u_{3}, v_{3}\right)$ on $\gamma_{2}^{*},\left(u_{3}^{*}, v_{3}^{*}\right)$ on $\gamma_{2}$, such that $\sigma\left(u_{3}^{*}, v_{3}^{*}, u, v\right)$ attain a local minimum at $\left(u_{3}, v_{3}\right)$, we then continue the curve starting at $\left(u_{3}, v_{3}\right)$ by $R_{2}\left(u_{3}, v_{3}\right)$.

If there is a point $\left(u_{3}, v_{3}\right)$ on $\gamma_{2}^{*}$ such that $\left(u_{3}^{*}, v_{3}^{*}\right)=\left(u_{1}, v_{1}\right)$, we then continue the curve starting at $\left(u_{3}, v_{3}\right)$ by $S_{2}\left(u_{0}, v_{0}\right)$. In fact, in this case we have $\sigma\left(u_{0}, v_{0} ; u_{1}, v_{1}\right)=\lambda_{1}\left(u_{1}, v_{1}\right)=\sigma\left(u_{1}, v_{1} ; u_{3}, v_{3}\right)$ which then implies that $\left(u_{3}, v_{3}\right) \in S_{2}\left(u_{0}, v_{0}\right)$ and $\sigma\left(u_{0}, v_{0} ; u_{3}, v_{3}\right)=\lambda_{2}\left(u_{1}, v_{1}\right)$. We have to check 
condition (E) for $\left\{\left(u_{0}, v_{0}\right) ;\left(u_{3}, v_{3}\right)\right\}$. Suppose condition (E) fails, then since $\left\{\left(u_{0}, v_{0}\right) ;\left(u_{1}, v_{1}\right)\right\}$ satisfies $(\mathrm{E})$, we would have a picture like the following:

$$
\sigma\left(u_{0}, v_{0} ; u, v\right)
$$

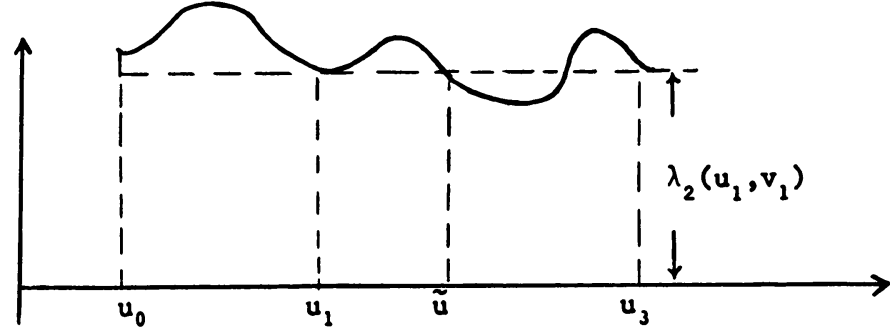

i.e. there is a point $(\tilde{u}, \tilde{v})$ on $S_{2}\left(u_{0}, v_{0}\right), u_{1}<\tilde{u}<u_{3}$, and $\sigma\left(u_{0}, v_{0} ; \tilde{u}, \widetilde{v}\right)=$ $\lambda_{2}\left(u_{1}, v_{1}\right)=\sigma\left(u_{0}, v_{0} ; u_{1}, v_{1}\right)$. The last equalities imply that $(\tilde{u}, \tilde{v}) \in S_{2}\left(u_{1}, v_{1}\right)$ and $\sigma\left(u_{1}, v_{1} ; u, v\right)=\lambda_{2}\left(u_{1}, v_{1}\right)$. But we have $\left(u_{1}, v_{1}\right)=\left(u_{3}^{*}, v_{3}^{*}\right)$; that is, $\left(u_{3}, v_{3}\right)$ is the first point on $S_{2}\left(u_{1}, v_{1}\right)$ with $\sigma\left(u_{1}, v_{1} ; u_{3}, v_{3}\right)=\lambda_{2}\left(u_{1}, v_{1}\right)$. This is a contradiction because $u_{1}<\tilde{u}<u_{3}$. So condition (E) is satisfied for $\left\{\left(u_{0}, v_{0}\right) ;\left(u_{3}, v_{3}\right)\right\}$. We have diagrams like the following:
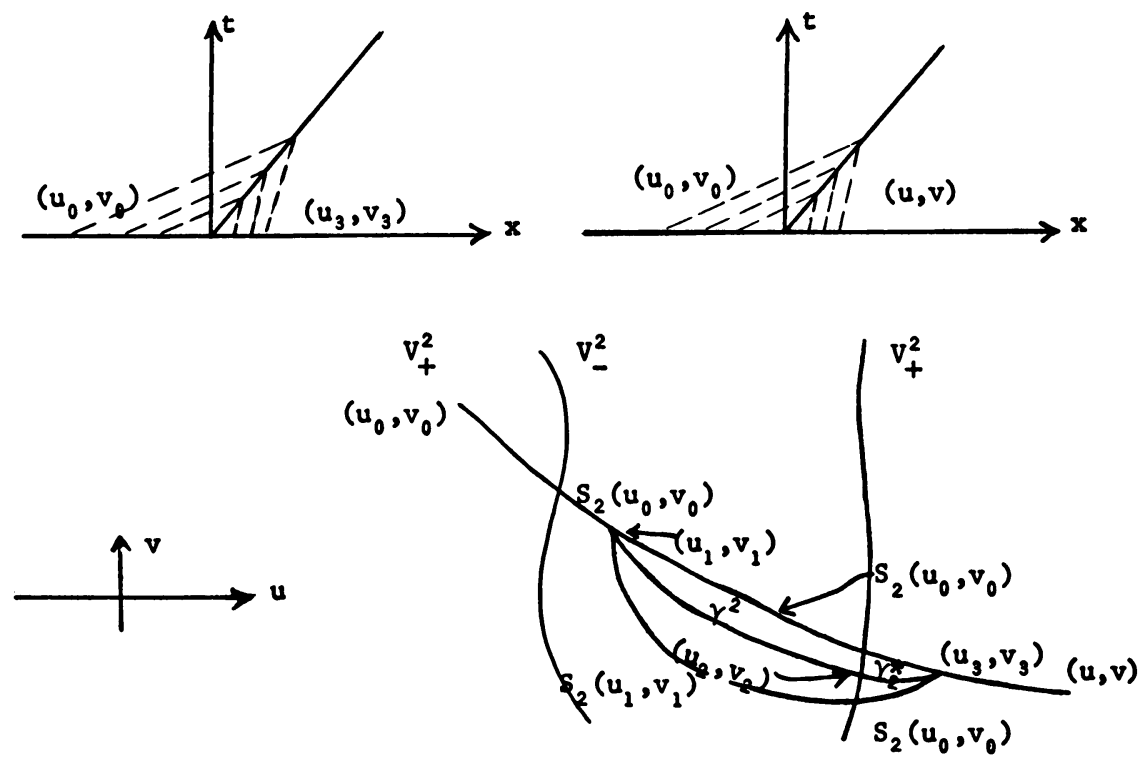

(ii) (a) For case (ii) (a) of Step 2:

We extend $S_{2}\left(u_{0}, v_{0}\right)$ until there exists a point $\left(u_{3}, v_{3}\right)$ which is the first point on $S_{2}\left(u_{0}, v_{0}\right), u_{3}>u_{2}$ such that $\sigma\left(u_{0}, v_{0} ; u_{3}, v_{3}\right)=\lambda_{2}\left(u_{3}, v_{3}\right)$ and that immediately to the right of $\left(u_{3}, v_{3}\right)$ along $R_{2}\left(u_{3}, v_{3}\right)$ is region $V_{-}^{2}$. Then as in case (i) of Step 2, we continue the curve from $\left(u_{3}, v_{3}\right)$ by $R_{2}\left(u_{3}, v_{3}\right)$ until it leaves region $V_{-}^{2}$.

(ii) (b) For case (ii) (b) of Step 2: 
We continue $R_{2}\left(u_{2}, v_{2}\right)$ until there exists a point $\left(u_{3}, v_{3}\right)$ at which $R_{2}\left(u_{2}, v_{2}\right)$ first leaves region $V_{-}^{2}$. Then we continue the curve by $\gamma_{2}^{*}$, where $\gamma_{2}$ is actually the curve $R_{2}\left(u_{2}, v_{2}\right)$ between $\left(u_{2}, v_{2}\right)$ and $\left(u_{3}, v_{3}\right)$. The diagrams look like the following:
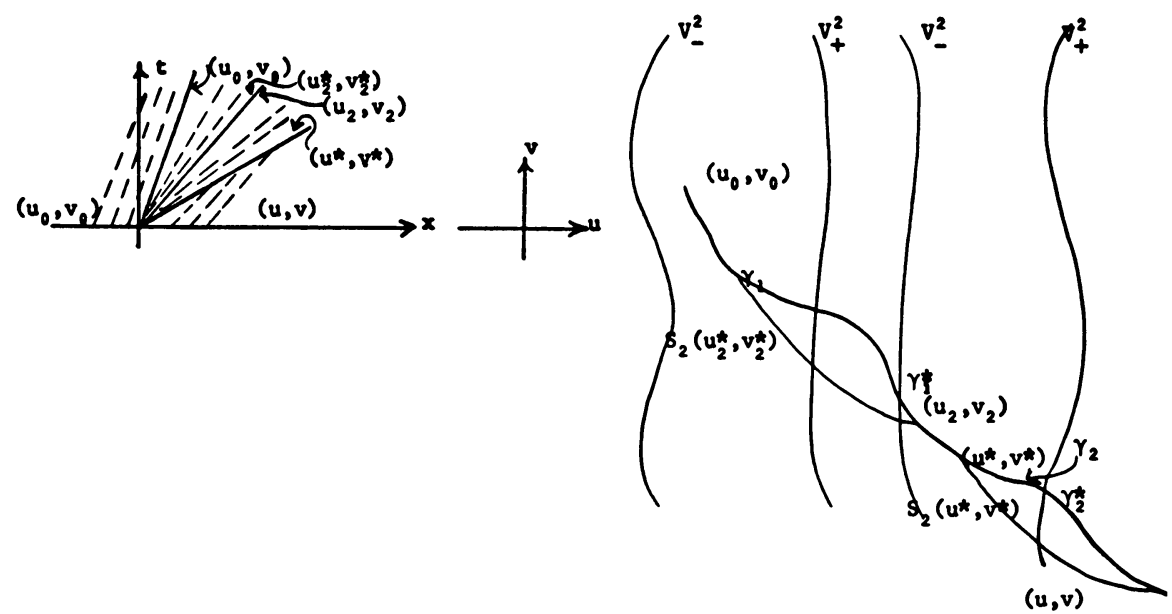

We need not describe how to continue the curve $\gamma$; it is analogous to the above steps. Thus the solution to the Riemann Problem $\left\{\left(u_{0}, v_{0}\right) ;(u, v)\right\}$ for $(u, v) \in$ $\boldsymbol{\gamma}$ takes the form

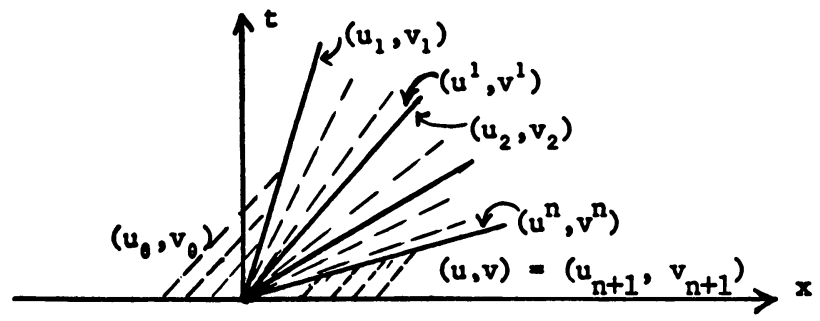

i.e. $\left\{\left(u_{0}, v_{0}\right) ;\left(u_{1}, v_{1}\right)\right\}$ and $\left\{\left(u^{n}, v^{n}\right) ;(u, v)\right\}$ are connected by one-sided contact discontinuities; $\left\{\left(u_{i}, v_{i}\right) ;\left(u^{i}, v^{i}\right)\right\}, 1 \leqslant i \leqslant n$, are connected by rarefaction waves and $\left\{\left(u^{i}, v^{i}\right) ;\left(u_{i+1}, v_{i+1}\right)\right\}, 1 \leqslant i \leqslant n-1$, are connected by two-sided contact discontinuities. Of course, we might have $\left(u_{1}, v_{1}\right)=\left(u^{n}, v^{n}\right)$ and $\left\{\left(u_{0}, v_{0}\right) ;(u, v)\right\}$ are connected by a single shock.

To prove that $\gamma_{i}^{*}$ is continuous and defined for $u$ and that condition (E) is satisfied for all discontinuities, we need some lemmas.

LEMMA 2.3. Let $\gamma$ be a curve with corresponding mixed curve $\gamma^{*}$, that is, $\gamma$ is a segment of an $R_{2}$ curve in the region $V_{-}^{2}$, and for every point $(u, v)$ on $\gamma^{*}$, there is a point $\left(u^{*}, v^{*}\right)$ on $\gamma$ such that $(u, v) \in S_{2}\left(u^{*}, v^{*}\right), u>u^{*}$, 
and $(u, v)$ is the first point on $S_{2}\left(u^{*}, v^{*}\right)$ such that $\sigma\left(u^{*}, v^{*} ; u, v\right)=\lambda_{2}(u, v)$. Then, along the curve $\gamma^{*}$,

$$
\frac{d v}{d u}=\frac{\left(u-u^{*}\right) g_{u}+\left(\lambda^{*}-f_{u}\right)\left(v-v^{*}\right)}{\left(v-v^{*}\right) f_{v}+\left(\lambda^{*}-g_{v}\right)\left(u-u^{*}\right)}
$$

where $g_{v}, f_{v}, f_{u}, g_{u}$ and $d v / d u$ are evaluated at $(u, v)$ and $\lambda^{*}=\lambda_{2}\left(u^{*}, v^{*}\right)$. Since $\lambda^{*}=\sigma$, we thus have $d v / d u=h_{2}\left(u^{*}, v^{*} ; u, v\right)$.

Proof. Use the Hugoniot condition for $\left\{\left(u^{*}, v^{*}\right) ;(u, v)\right\}$ along with equalities:

$$
\begin{gathered}
\frac{d v^{*}}{d u^{*}}=a^{*} \equiv a_{2}\left(u^{*}, v^{*}\right), \quad \frac{d v}{d u}=\frac{v_{1}+v_{2} d v^{*} / d u^{*}}{u_{1}+u_{2} d v^{*} / d u^{*}} \\
u_{1}=\left.\frac{\partial u}{\partial u^{*}}\right|_{(u, v)}, \quad u_{2}=\left.\frac{\partial u}{\partial v^{*}}\right|_{(u, v)}, \text { etc. }
\end{gathered}
$$

LEMMA 2.4. Let $\left(u_{1}^{*}, v_{1}^{*}\right) \in \gamma,\left(u_{2}^{*}, v_{2}^{*}\right) \in \gamma,\left(u_{1}, v_{1}\right) \in \gamma^{*},\left(u_{2}, v_{2}\right) \in \gamma^{*}$, and $u_{1}^{*}>u_{2}^{*}$, then $u_{2}>u_{1}$.

Proof. We have only to prove the lemma when $\left|u_{1}^{*}-u_{2}^{*}\right|$ is small. Suppose, otherwise, we have $u_{2}<u_{1}$, and the picture looks like:

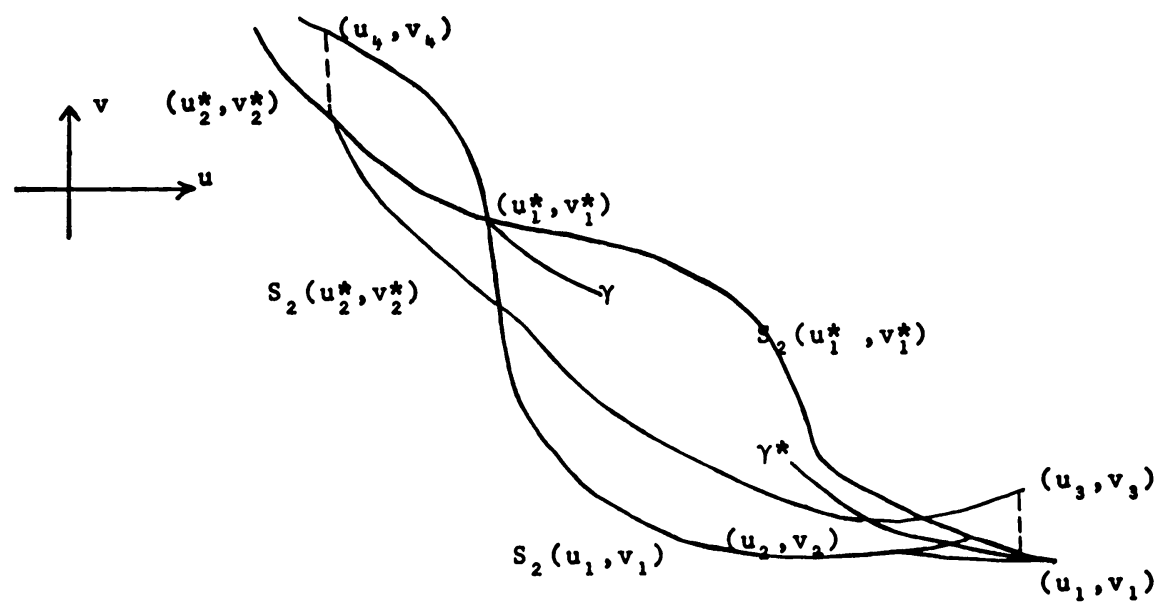

By continuity of $\sigma$, we have $\left|u_{1}-u_{2}\right|$ small. Pick points $\left(u_{3}, v_{3}\right) \in S_{2}\left(u_{2}^{*}, v_{2}^{*}\right)$ and $\left(u_{4}, v_{4}\right) \in S_{2}\left(u_{1}, v_{1}\right), u_{3}=u_{1}, u_{4}=u_{2}^{*}$. By Lemma 2.3, we know $S_{2}\left(u_{2}^{*}, v_{2}^{*}\right)$ is tangent to $\gamma^{*}$ at $\left(u_{2}, v_{2}\right)$; thus $\left|v_{3}-v_{1}\right|=O_{2}\left|u_{1}-u_{2}\right|$. By Lemma 2.3 and continuity of $\sigma$, we have $O_{2}\left|u_{1}-u_{2}\right|=O_{2}\left|u_{1}^{*}-u_{2}^{*}\right|$. So $\left|v_{3}-v_{1}\right|=O_{2}\left|u_{1}^{*}-u_{2}^{*}\right|$. The definition of $\gamma^{*}$ gives $\sigma\left(u_{1}, v_{1} ; u_{1}^{*}, v_{1}^{*}\right)=$ $\lambda_{2}\left(u_{1}^{*}, v_{1}^{*}\right)$, and thus, by Lemma $1.5, h_{2}\left(u_{1}, v_{1} ; u_{1}^{*}, v_{1}^{*}\right)=a_{2}\left(u_{1}^{*}, v_{1}^{*}\right)$, so $\left|v_{4}-v_{2}^{*}\right|=O_{2}\left|u_{1}^{*}-u_{2}^{*}\right|$. 
The last equality along with $\left|v_{3}-v_{1}\right|=O_{2}\left|u_{1}^{*}-u_{2}^{*}\right|$ gives $\sigma\left(u_{1}, v_{1} ; u_{4}, v_{4}\right)=$ $\sigma\left(u_{3}, v_{3} ; u_{2}^{*}, v_{2}^{*}\right)+O_{2}\left|u_{1}^{*}-u_{2}^{*}\right|$.

Since condition (E) is satisfied for the pair $\left\{\left(u_{2}^{*}, v_{2}^{*}\right) ;\left(u_{2}, v_{2}\right)\right\}$, we know that $d \sigma / d \mu \leqslant 0$ along $S_{2}\left(u_{2}^{*}, v_{2}^{*}\right)$ at the point $\left(u_{2}, v_{2}\right)$. This implies that $\sigma\left(u_{2}^{*}, v_{2}^{*} ; u_{2}, v_{2}\right) \geqslant \sigma\left(u_{2}^{*}, v_{2}^{*} ; u_{3}, v_{3}\right)+O_{2}\left|u_{1}-u_{2}\right|$.

Since $\sigma\left(u_{1}, v_{1} ; u_{1}^{*}, v_{1}^{*}\right)=\lambda_{2}\left(u_{1}^{*}, v_{1}^{*}\right)$, we have, by Lemma $1.4, d \sigma / d \mu=0$ along $S_{2}\left(u_{1}, v_{1}\right)$ at $\left(u_{1}^{*}, v_{1}^{*}\right)$; thus $\sigma\left(u_{1}, v_{1} ; u_{1}^{*}, v_{1}^{*}\right)=\sigma\left(u_{1}, v_{1} ; u_{4}, v_{4}\right)+$ $O_{2}\left|u_{1}-u_{2}\right|$.

Since $\gamma \subset V_{-}^{2}$, we have $\lambda_{2}\left(u_{1}^{*}, v_{1}^{*}\right) \geqslant \lambda_{2}\left(u_{2}^{*}, v_{2}^{*}\right)+k\left|u_{1}-u_{2}\right|$ for some $k>0$.

Using the inequalities just derived, we have

$$
\begin{aligned}
\lambda_{2}\left(u_{2}^{*}, v_{2}^{*}\right) & =\sigma\left(u_{2}^{*}, v_{2}^{*} ; u_{2}, v_{2}\right) \geqslant \sigma\left(u_{2}^{*}, v_{2}^{*} ; u_{3}, v_{3}\right)+O_{2}\left|u_{1}^{*}-u_{2}^{*}\right| \\
& =\sigma\left(u_{1}, v_{1} ; u_{4}, v_{4}\right)+O_{2}\left|u_{1}^{*}-u_{2}^{*}\right| \\
& =\sigma\left(u_{1}, v_{1} ; u_{1}^{*}, v_{1}^{*}\right)+O_{2}\left|u_{1}^{*}-u_{2}^{*}\right| \\
& =\lambda_{2}\left(u_{1}^{*}, v_{1}^{*}\right)+O_{2}\left|u_{1}^{*}-u_{2}^{*}\right| \\
& \geqslant \lambda_{2}\left(u_{2}^{*}, v_{2}^{*}\right)+k\left|u_{1}^{*}-u_{2}^{*}\right|+O_{2}\left|u_{1}^{*}-u_{2}^{*}\right|, \quad k>0,
\end{aligned}
$$

which is a contradiction. The lemma is proved. Q.E.D.

Similarly, we can prove

LEMMA 2.5. Let $\left(u_{1}^{*}, v_{1}^{*}\right) \in \gamma,\left(u_{1}, v_{1}\right) \in \gamma^{*}$, and suppose that $\sigma\left(u_{1}^{*}, v_{1}^{*} ; u, v\right)$ attains a local minimum at $\left(u_{1}, v_{1}\right)$. Then, for $\left(u^{*}, v^{*}\right) \in \gamma$, $\left|u^{*}-u_{1}^{*}\right|$ small, we have

(i) There is no point $(u, v) \in S_{2}\left(u^{*}, v^{*}\right),\left|u-u_{1}\right|$ small or $u^{*}<u \leqslant u_{1}$, so that condition (E) is satisfied for $\left\{\left(u^{*}, v^{*}\right) ;(u, v)\right\}$, provided $u^{*}<u_{1}^{*}$.

(ii) There is point $(u, v) \in S_{2}\left(u^{*}, v^{*}\right),\left|u-u_{1}\right|$ small, $u^{*}<u \leqslant u_{1}$, so that condition (E) is satisfied for $\left\{\left(u^{*}, v^{*}\right) ;(u, v)\right\}$, provided $u^{*}>u_{1}^{*}$.

Using Lemmas 2.4 and 2.5 , we can prove

LEMMA 2.6. Let $\left(u_{3}, v_{3}\right) \in S_{2}\left(u_{1}, v_{1}\right), u_{2}>u_{1}, \sigma\left(u_{1}, v_{1} ; u_{3}, v_{3}\right)=$ $\lambda_{2}\left(u_{1}, v_{1}\right)$ and suppose that $\left\{\left(u_{1}, v_{1}\right) ;\left(u_{3}, v_{3}\right)\right\}$ satisfies (E). Take any $\left(u^{*}, v^{*}\right) \in$ $R_{2}\left(u_{1}, v_{1}\right), u^{*}<u_{1}$, such that any point on $R_{2}\left(u_{1}, v_{1}\right)$ between $\left(u^{*}, v^{*}\right)$ and $\left(u_{1}, v_{1}\right)$ lies in region $V_{-}^{2}$. Then there is no point $(u, v)$ on $S_{2}\left(u^{*}, v^{*}\right), u^{*}<$ $u \leqslant u_{3}$, such that condition (E) is satisfied for $\left\{\left(u^{*}, v^{*}\right) ;(u, v)\right\}$.

REMARK. Part (i) of Lemma 2.5 implies that the curve $\gamma^{*}$ cannot be extended beyond $\left(u_{1}, v_{1}\right)$; and part (ii) implies that there are points on $\gamma^{*}$ left of $\left(u_{1}, v_{1}\right)$. Lemma 2.6 extends the results globally.

Using Lemmas $2.4,2.5$ and 2.6 , we finally have 
Lemma 2.7. Any mixed curve is a smooth and decreasing function of $u$.

We make the following assumption:

$$
\left\{\begin{array}{l}
\text { Either (i) } g_{u} \text { is finite for finite } u, \\
\text { or (ii) the integral curve of } d v / d u=g_{u} \text { does not escape along any } \\
\text { vertical line and every finite-width vertical zone contains only finite } \\
\text { many curves in } V_{0}^{2} .
\end{array}\right.
$$

THEOREM 2.1. Under the assumptions (0.4), (0.5), (2.1) and (2.2), the curve $\gamma\left(u_{0}, v_{0}\right)$ constructed is defined for all $u$ and is a decreasing function of $u$. Here $\gamma\left(u_{0}, v_{0}\right)$ is such that points $(u, v)$ on $\gamma\left(u_{0}, v_{0}\right)$ can be connected to $\left(u_{0}, v_{0}\right)$ on the right by forward waves.

Proof. The fact that the curve $\boldsymbol{\gamma}$ is a decreasing function of $u$ is obvious by now. We have only to show that $\gamma$ does not have vertical asymptotes. With Lemma 1.1 and Lemma 2.3, we need only to treat the cases when $\gamma$ is composed of a single rarefaction curve $R_{2}(\tilde{u}, \widetilde{v})$ or when $\gamma$ is composed of infinitely many mixed and rarefaction curves eventually. In the first case, $\lambda_{2}$ is increasing along $R_{2}(\tilde{u}, \tilde{v})$, and thus $d v / d u=a_{2}=g_{u} / \lambda-g_{v}<g_{u} / \lambda_{2}(u, v)<$ $g_{u} / \lambda_{2}(\tilde{u}, \tilde{v})$. By $(2.2)$, we know $R(\tilde{u}, \tilde{v})$ does not have vertical asymptote. In the case $\gamma$ is composed of infinitely many rarefaction and mixed curves, and $\boldsymbol{\gamma}$ has vertical asymptote, we show this is impossible by using (2.2). From (ii) of (2.2), this cannot happen, since the intersection of a rarefaction curve with the adjacent mixed curve is a point in $V_{0}^{2}$. We treat the case when (i) of (2.2) is assumed. With Lemma 1.1, we may assume that $S_{2}\left(u_{0}, v_{0}\right)$ does not appear in $\gamma$ eventually, thus the solution to the Riemann Problem $\left\{\left(u_{0}, v_{0}\right) ;(u, v)\right\}$, $u$ close to $\bar{u}$ and $(u, v) \in \gamma$ is of the form that $\left(u_{0}, v_{0}\right)$ is connected to a fixed $\left(u_{1}, v_{1}\right)$ on $\gamma$ by a contact discontinuity and $\left(u_{1}, v_{1}\right)$ is connected to $(u, v)$ by contact discontinuities and rarefaction waves. With the preceding argument, in order to have $\gamma$ escape along $u=\bar{u}$, there must be sequences $\left\{\left(u_{n}\right.\right.$, $\left.\left.u_{n}\right)\right\}$ and $\left\{\left(u^{n}, v^{n}\right)\right\}$ on $\gamma$, such that $\left(u_{n}, v_{n}\right)$ is connected to $\left(u^{n}, v^{n}\right)$ by a two-sided contact discontinuity and $\lim \left(v_{n}-v^{n}\right) /\left(u_{n}-u^{n}\right)=-\infty$. However, since $g_{v} \leqslant 0, g_{u}<0$, the last equality implies

$$
\begin{aligned}
\sigma\left(u^{n}, v^{n} ; u_{n}, v_{n}\right) & =\frac{g\left(u^{n}, v^{n}\right)-g\left(u_{n}, v_{n}\right)}{v^{n}-v_{n}}<\frac{g\left(u^{n}, v_{n}\right)-g\left(u_{n}, v_{n}\right)}{v^{n}-v_{n}} \\
& =\frac{\left(u^{n}-u_{n}\right) g_{u}\left(\tilde{u}, v_{n}\right)}{v^{n}-v_{n}} \rightarrow 0 \quad \text { as } u_{n} \rightarrow \bar{u} .
\end{aligned}
$$


But since

$$
\sigma\left(u^{n}, v^{n} ; u_{n}, v_{n}\right)>\lambda_{2}\left(u_{1}, v_{1}\right)>0,
$$

this is a contradiction. We have finished the proof of the theorem. Q.E.D.

Similarly, under the assumptions (0.4), (0.5), (2.1) and the following:

The integral curve of $d v / d u=a$ does not have horizontal asymptote (2.3) and any finite-width horizontal zone contains only finite many curves in $V_{0}^{1}$,

we have

THEOREM 2.2. Given any $\left(u_{0}, v_{0}\right) \in U$, there is a curve $\beta\left(u_{0}, v_{0}\right)=\beta$ defined for all $v$ and contained in the quadrants $\left\{(u, v) \mid u \geqslant u_{0}, v \geqslant v_{0}\right\}$ and $\left\{(u, v) \mid u \leqslant u_{0}, v \leqslant v_{0}\right\}$ such that point $(u, v)$ on $\beta$ can be connected to $\left(u_{0}, v_{0}\right)$ on the right by forward shock, rarefaction waves and contact discontinuities.

From Theorems 2.1 and 2.2, we finally have the following theorem.

THEOREM 2.3. Suppose that (0.4), (0.5), (2.1), (2.2) and (2.3) hold. Then the Riemann Problem $\left\{\left(u_{l}, v_{l}\right) ;\left(u_{r}, v_{r}\right)\right\}$ for general data can be solved in the class of shocks, rarefaction waves and contact discontinuities, and condition (E) is satisfied across discontinuities.

Proof. Divide the region $U$ into four quadrants as shown in the diagram that follows

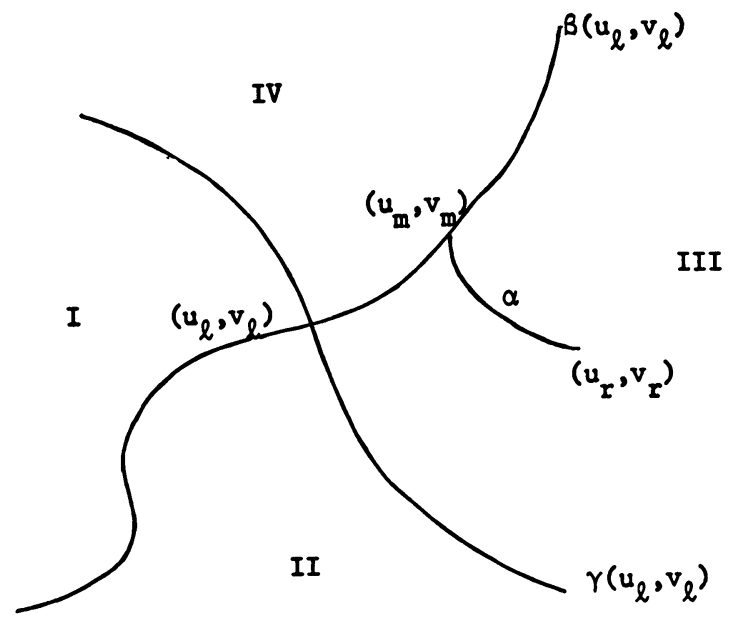


Suppose, say, $\left(u_{r}, v_{r}\right) \in$ III. Construct a curve $\alpha\left(u_{r}, v_{r}\right)$ from $\left(u_{r}, v_{r}\right)$ such that a point $(u, v)$ on $\alpha$ can be connected to $\left(u_{r}, v_{r}\right)$ on the left by forward waves. An analogy of Theorems 2.1 and 2.2 yields that $\alpha$ is defined for all $u<$ $u_{r}$. Since $\beta$ is defined for all $v, \alpha$ intercepts $\beta$ at some point $\left(u_{m}, v_{m}\right)$. We then solve the Riemann Problem $\left\{\left(u_{l}, v_{l}\right) ;\left(u_{r}, v_{r}\right)\right\}$ by connecting $\left(u_{l}, v_{l}\right)$ to $\left(u_{m}, v_{m}\right)$ by backward waves and $\left(u_{m}, v_{m}\right)$ to $\left(u_{r}, v_{r}\right)$ by forward waves, as shown in the picture below

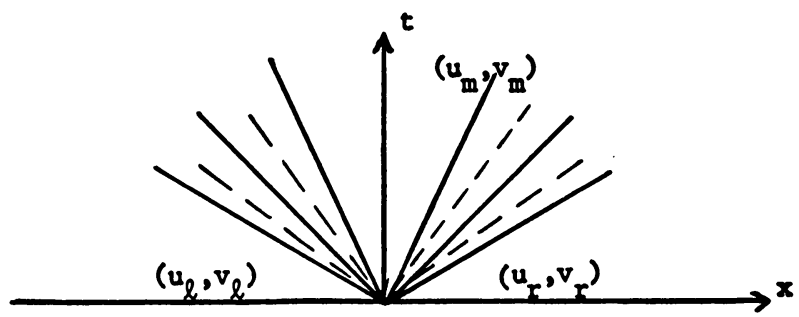

This completes the proof of the theorem. Q.E.D.

3. Uniqueness. In this section we shall prove that the solution to the Riemann Problem is unique, provided condition (E) is satisfied.

THEOREM 3.1. Under the assumptions (0.4), (0.5) and (2.1), if $\left(u_{l}, v_{l}\right)$ is connected to $\left(u_{r}, v_{r}\right)$ on the right by finite number forward shocks, rarefaction waves and contact discontinuities and condition $(\mathrm{E})$ is satisfied across discontinuities, then $\left(u_{r}, v_{r}\right) \in \gamma\left(u_{l}, v_{l}\right)$ and the solution is the one constructed in $\S 2$.

Proof. By simple geometric consideration, using Lemma 1.7, we know that the solution must be of the form

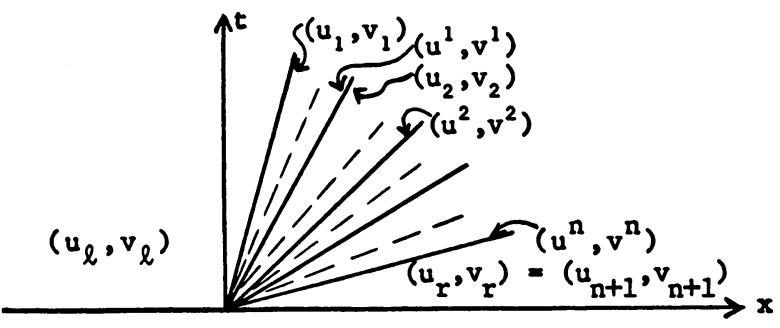

where $\left\{\left(u^{i}, v^{i}\right) ;\left(u_{i+1}, v_{i+1}\right)\right\}, 1 \leqslant i \leqslant n-1$, are connected by contact discontinuities, $\left\{\left(u_{i}, v_{i}\right) ;\left(u^{i}, v^{i}\right)\right\}, 1 \leqslant i \leqslant n-1$, are connected by rarefaction waves, and $\left\{\left(u_{l}, v_{l}\right) ;\left(u_{1}, v_{1}\right)\right\}$ and $\left\{\left(u^{n}, v^{n}\right) ;\left(u_{r}, v_{r}\right)\right\}$ are connected by shocks or contact discontinuities. 
We assume that $u^{i} \neq u_{i+1}$, that is, there is a real discontinuity between $\left(u^{i}, v^{i}\right)$ and $\left(u_{i+1}, v_{i+1}\right)$. Suppose that $u_{1}>u_{l}$. The case $u_{1} \leqslant u_{l}$ can be treated similarly. The proof is based on several claims.

ClaIm 1. $\left(u_{i}, v_{i}\right) \in V_{-}^{2}$ and $u_{i}<u^{i}, u^{i}<u_{i+1}, i=1,2, \cdots, n$.

Proof. We shall show that $\left(u_{1}, v_{1}\right) \in V_{+}^{2} \cup V_{0}^{2}$ is impossible.

If $\left(u_{1}, v_{1}\right) \in V_{+}^{2}$, then since $\sigma\left(u_{l}, v_{l} ; u_{1}, v_{1}\right)=\lambda_{2}\left(u_{1}, v_{1}\right)$, we have, by Lemma 1.6, that $\sigma\left(u_{l}, v_{l} ; u, v\right)$ has a local maximum at $\left(u_{1}, v_{1}\right)$. This contradicts the condition (E) for $\left\{\left(u_{l}, v_{l}\right) ;\left(u_{1}, v_{1}\right)\right\}$.

If $\left(u_{1}, v_{1}\right) \in V_{0}^{2}$, and immediately to the left of $\left(u_{1}, v_{1}\right)$ is region $V_{+}^{2}$, then, by Lemma 2.2, $\sigma\left(u_{l}, v_{l} ; u, v\right)$ is increasing at $\left(u_{1}, v_{1}\right)$. This again contradicts condition (E) for $\left\{\left(u_{l}, v_{l}\right) ;\left(u_{1}, v_{1}\right)\right\}$.

If $\left(u_{1}, v_{1}\right) \in V_{0}^{2}$, and immediately to the left of $\left(u_{1}, v_{1}\right)$ is region $V_{-}^{2}$, then, by Lemma 2.1 , immediately to the right of $\left(u_{1}, v_{1}\right)$ is region $V_{+}^{2}$. However, we have $\left(u^{1}, v^{1}\right) \in R_{2}\left(u_{1}, v_{1}\right)$ and $\left\{\left(u_{1}, v_{1}\right) ;\left(u^{1}, v^{1}\right)\right\}$ are connected by forward rarefaction wave. This implies that, if $u^{1}>u_{1}$, then the $R_{2}$ curve between $\left(u_{1}, v_{1}\right)$ and $\left(u^{1}, v^{1}\right)$ lies in $V_{-}^{2}$, and, if $u^{1}<u_{1}$, then the $R_{2}$ curve between $\left(u_{1}, v_{1}\right)$ and $\left(u^{1}, v^{1}\right)$ lies in $V_{+}^{2}$. In our present case, this is impossible.

In any case, we have $\left(u_{1}, v_{1}\right) \in V_{-}^{2}$. Thus, by Lemma 1.3 , since $\left\{\left(u^{1}, v^{1}\right)\right.$; $\left.\left(u_{2}, v_{2}\right)\right\}$ satisfies condition $(\mathrm{E})$, we know that $u_{2}>u^{1}$. It is clear that Claim 1 can be proved by induction.

Claim 2. (i) Let $\hat{u}^{i-1}=\max \left\{u \mid(u, v) \in S_{2}\left(u^{i-1}, v^{i-1}\right), u^{i-1} \leqslant u \leqslant u_{r}\right.$, $\left\{\left(u^{i-1}, v^{i-1}\right) ;(u, v)\right\}$ satisfies $\left.(\mathrm{E})\right\}, 1 \leqslant i \leqslant n+1$; then $u_{i}=\hat{u}^{i-1}$.

(ii) Let $(u, v) \in R_{2}\left(u_{i}, v_{i}\right) ;(\tilde{u}, \tilde{v}) \in S_{2}(u, v), u_{i} \leqslant u \leqslant u^{i}, u<\tilde{u} \leqslant u_{r}$; then $\{(u, v) ;(\widetilde{u}, \widetilde{v})\}$ does not satisfy $(\mathrm{E}), 1 \leqslant i \leqslant n$.

Proof (by induction). Part (i) is clearly true for $i=n+1$. By Lemma 2.6, (ii) is true for $i=n$. Suppose that (i) fails for $i=n$. Pick $(\bar{u}, \bar{v}) \in$ $S_{2}\left(u^{n-1}, v^{n-1}\right), u_{n}<\bar{u} \leqslant u_{r}$, such that $\sigma\left(u^{n-1}, v^{n-1} ; \bar{u}, \bar{v}\right)=\lambda_{2}\left(u^{n-1}, v^{n-1}\right)$. Since, by Claim $1,\left(u_{n}, v_{n}\right) \in V_{-}^{2}$, we know that $\sigma\left(u^{n-1}, v^{n-1} ; u, v\right)$ attains a local minimum at $\left(u_{n}, v_{n}\right)$ and $\sigma\left(u^{n-1}, v^{n-1} ; u_{n}, v_{n}\right)=\lambda_{2}\left(u^{n-1}, v^{n-1}\right)=$ $\lambda_{2}\left(u_{n}, v_{n}\right)$. The last equalities together with the fact that $o\left(u^{n-1}, v^{n-1} ; \bar{u}, \bar{v}\right)=$ $\lambda_{2}\left(u^{n-1}, v^{n-1}\right)$ implies that $(\bar{u}, \bar{v}) \in S_{2}\left(u_{n}, v_{n}\right)$ and $\sigma\left(u_{n}, v_{n} ; \bar{u}, \bar{v}\right)=$ $\lambda_{2}\left(u_{n}, v_{n}\right)$.

Since $\left(u_{n}, v_{n}\right) \in V_{-}^{2}$, we have $d \sigma / d \mu>0$ along $S_{2}\left(u_{n}, v_{n}\right)$ at points close to $\left(u_{n}, v_{n}\right)$. Thus there exists $(u, v) \in S_{2}\left(u_{n}, v_{n}\right), u_{n}<u \leqslant \bar{u}$, such that $\left\{\left(u_{n}, v_{n}\right),(u, v)\right\}$ satisfies (E). 


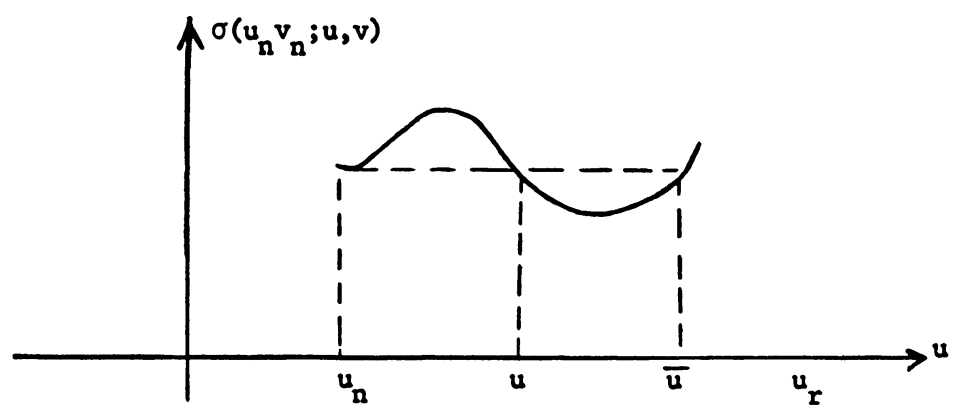

This contradicts (ii) of this claim for $i=n$. Thus (i) holds tor $i=n$. We then prove (ii) for $i=n-1$ by using Lemmas 2.5 and 2.6. This completes the proof of Claim 2 by induction.

We now return to the proof of Theorem 3.1.

Claim 2, (i) implies that $u_{1}=\hat{u}_{l}$. Since $\left(u_{1}, v_{1}\right) \in S_{2}\left(u_{l}, v_{l}\right)$, we know that $\left(u_{1}, v_{1}\right)$ is uniquely determined by $\left(u_{l}, v_{l}\right)$ and $u_{r}$.

Suppose that there exists $\left(\bar{u}^{i}, \bar{v}^{i}\right)$, and $\left(\bar{u}_{i}, \bar{v}_{i}\right), i=1,2, \cdots, \bar{u}^{1} \neq u^{1}$, and that

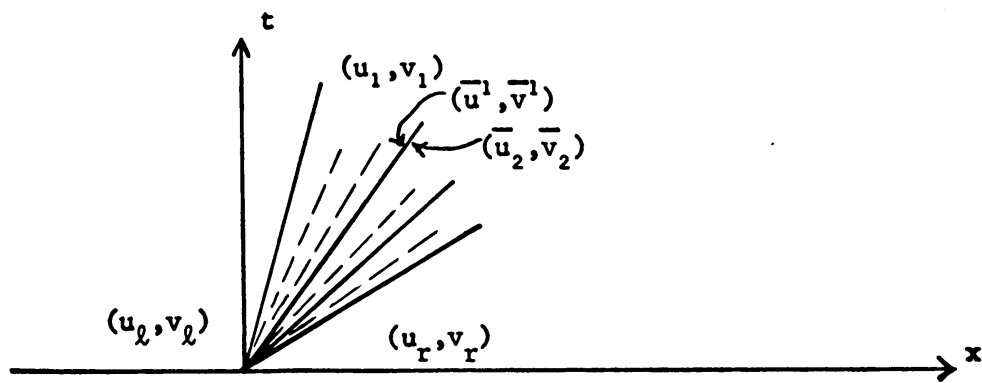

solves the Riemann Problem $\left\{\left(u_{l}, v_{l}\right) ;\left(u_{r}, v_{r}\right)\right\}$. Without loss of generality, assume $\bar{u}^{1}<u^{1}$. By Claim $1, \bar{u}^{1}<\bar{u}_{2} \leqslant u_{r}$ and $\left\{\left(\bar{u}^{1}, \bar{v}^{1}\right) ;\left(\bar{u}_{2}, \bar{v}_{2}\right)\right\}$ satisfies condition (E). This contradicts part (ii) of Claim 2 for $i=1$. Thus $\bar{u}^{1}=u^{1}$. Since $\left(u^{1}, v^{1}\right) \in R_{2}\left(u_{1}, v_{1}\right)$, it follows that $\left(u^{1}, v^{1}\right)$ is uniquely determined by $\left(u_{l}, v_{l}\right)$ and $u_{r}$. The proof of Theorem 3.1 is complete by induction. Q.E.D.

Theorem 3.2. Assume (0.4), (0.5), (2.1), (2.2) and (2.3). Take $\left(u_{0}, v_{0}\right) \in$ $U,\left(u_{1}, v_{1}\right)$ and $\left(u_{2}, v_{2}\right)$ on $\beta\left(u_{0}, v_{0}\right), v_{1} \neq v_{2}$. Then $\gamma\left(u_{1}, v_{1}\right) \cap \gamma\left(u_{2}, v_{2}\right) \neq$ $\varnothing$. Here $\beta$ and $\gamma$ are defined in Theorems 2.1 and 2.2.

Proof. Suppose, otherwise, there is $\left(u_{3}, v_{3}\right) \in \gamma\left(u_{1}, v_{1}\right) \cap \gamma\left(u_{2}, v_{2}\right)$. Assume that $u_{3}>u_{1}, u_{3}>u_{2}$. The case $u_{3}<u_{1}, u_{3}<u_{2}$ can be treated similarly.

Choose $\left(u^{1}, v^{1}\right) \in \beta\left(u_{0}, v_{0}\right),\left(u^{1}, v^{1}\right)$ lying between $\left(u_{1}, v_{1}\right)$ and $\left(u_{2}, v_{2}\right)$. Then $\left(u^{1}, v^{1}\right)$ intercepts $\gamma\left(u_{i}, v_{i}\right), i=1$ or 2 , at, say $\left(\bar{u}^{1}, \bar{v}^{1}\right), u^{1}<\bar{u}^{1} \leqslant u_{3}$. 
Next choose $\left(u^{2}, v^{2}\right)$ on $\beta\left(u_{0}, v_{0}\right)$ between $\left(u^{1}, v^{1}\right)$ and $\left(u_{i}, v_{i}\right)$. Then $\gamma\left(u^{2}, v^{2}\right)$ intercepts $\gamma\left(u_{i}, v_{i}\right)$ or $\gamma\left(u^{1}, v^{1}\right)$ at say $\left(\bar{u}^{2}, \bar{v}^{2}\right), u^{2}<\bar{u}^{2} \leqslant u_{3}$. Continuing the process, we then get a sequence $\left\{\left(u^{i}, v^{i}\right)\right\}$. Without introducing a new notation, by passing to a subsequence, we may let $\left(u_{1}, v_{1}\right)$ be the limit point of the sequence $\left\{\left(u^{i}, v^{i}\right)\right\}$. Thus we can assume that $\left(u_{2}, u_{2}\right)$ is arbitrarily close to $\left(u_{1}, v_{1}\right)$. We consider only the case $v_{1} \geqslant v_{0}, v_{2} \geqslant v_{0}$. The other cases can be treated similarly.

Let $\alpha\left(u_{3}, v_{3}\right)$ be a curve such that any point $(u, v)$ on $\alpha\left(u_{3}, v_{3}\right)$ can be connected to $\left(u_{3}, v_{3}\right)$ on the left by forward waves. An analogue of Theorem 3.1 says that $\left(u_{2}, v_{2}\right)$ and $\left(u_{1}, v_{1}\right)$ are both on $\alpha\left(u_{3}, v_{3}\right)$ and that $\alpha\left(u_{3}, v_{3}\right)$ is composed of shock, rarefaction and mixed curves.

By choosing $\left(u_{2}, v_{2}\right)$ close enough to $\left(u_{1}, v_{1}\right)$, we have

$$
\begin{aligned}
\text { either }\left.\frac{d u}{d v}\right|_{\alpha\left(u_{3}, u_{3}\right)} \geqslant\left.\frac{d v}{d u}\right|_{\beta\left(u_{0}, v_{0}\right)} \geqslant 0 \text { at }\left(u_{2}, v_{2}\right) \\
\quad \text { or }\left.\frac{d v}{d u}\right|_{\alpha\left(u_{3}, v_{3}\right)} \leqslant\left.\frac{d v}{d u}\right|_{\beta\left(u_{0}, v_{0}\right)} \leqslant 0 \text { at }\left(u_{2}, v_{2}\right) .
\end{aligned}
$$

We only prove the theorem for the second case; i.e. we assume (3.1). The first case can be treated similarly.

Since $a_{1}>0$, by (3.1), we have $d v /\left.d u\right|_{\beta\left(u_{0}, v_{0}\right)} \neq a_{1}$ at $\left(u_{2}, v_{2}\right)$. Hence, by the construction of $\beta\left(u_{0}, v_{0}\right)$, there exists $\left(u^{0}, v^{0}\right) \in \beta\left(u_{0}, v_{0}\right)$ such that $\left\{\left(u^{0}, v^{0}\right) ;\left(u_{2}, v_{2}\right)\right\}$ satisfies (E) and $d v /\left.d u\right|_{\beta\left(u_{0}, v_{0}\right)}=h_{2}\left(u^{0}, v^{0} ; u_{2}, v_{2}\right)$ at $\left(u_{2}, v_{2}\right)$.

If $d u /\left.d v\right|_{\alpha\left(u_{3}, v_{3}\right)}=a_{2}$ at $\left(u_{2}, v_{2}\right)$, then by (3.1), $0>h\left(u^{0}, v^{0} ; u_{2}, v_{2}\right) \geqslant$ $a_{2}\left(u_{2}, v_{2}\right)$. This would then lead to $\sigma-\lambda_{2}>0$, which is a contradiction, since $\sigma<0$ and $\lambda_{2}>0$. So there exists $\left(u^{3}, v^{3}\right)$ on $\alpha\left(u_{3}, v_{3}\right)$ such that $\left\{\left(u_{2}, v_{2}\right) ;\left(u^{3}, v^{3}\right)\right\}$ satisfies (E) and $d v /\left.d u\right|_{\alpha_{\left(u_{3}, v_{3}\right)}}=h_{2}\left(u^{3}, v^{3} ; u_{2}, v_{2}\right)$ at $\left(u_{2}, v_{2}\right)$. (3.1) then gives

$$
h_{2}\left(u^{3}, v^{3} ; u_{2}, v_{2}\right) \leqslant h_{2}\left(u^{0}, v^{0} ; u_{2}, v_{2}\right) \leqslant 0 .
$$

By condition (E) for $\left\{\left(u_{2}, v_{2}\right) ;\left(u^{3}, v^{3}\right)\right\}$ and $\left\{\left(u^{0}, v^{0}\right) ;\left(u_{2}, v_{2}\right)\right\}$, using Lemmas $1.4,1.5$ and 1.7 , we have

$$
\begin{aligned}
& \frac{d \sigma}{d \mu}>0 \text { along } S_{2}\left(u^{3}, v^{3}\right) \text { at }\left(u_{2}, v_{2}\right), \\
& \frac{d \sigma}{d \mu}<0 \text { along } S_{1}\left(u^{0}, v^{0}\right) \text { at }\left(u_{2}, v_{2}\right) .
\end{aligned}
$$


Pick points $\left(u_{4}, v_{4}\right)$ on $S_{1}\left(u^{0}, v^{0}\right)$ and $\left(u_{5}, v_{4}\right)$ on $S_{2}\left(u^{3}, v^{3}\right)$, $\left|v_{4}-v_{2}\right|$ small, $v_{4}>v_{2}$. We have the following picture:

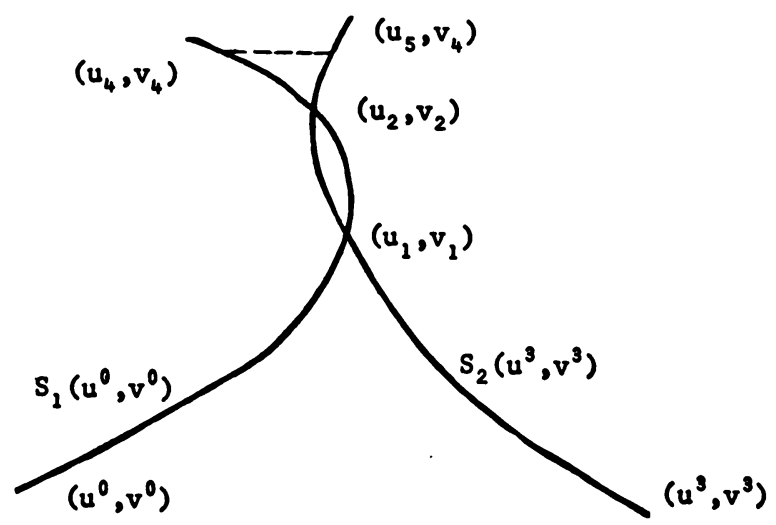

By (3.2), we know

$$
u_{5} \geqslant u_{4}+O_{2}\left|v_{4}-v_{2}\right|
$$

Since $g_{u}<0,(3.5)$ implies

$$
g\left(u_{5}, v_{4}\right) \leqslant g\left(u_{4}, v_{4}\right)+O_{2}\left|v_{4}-v_{2}\right| .
$$

Since $\sigma=\Delta g / \Delta u,(3.3)$ implies that

$$
\frac{g\left(u_{5}, v_{4}\right)-g\left(u^{3}, v^{3}\right)}{v_{4}-v^{3}}>\frac{g\left(u_{2}, v_{2}\right)-g\left(u^{3}, v^{3}\right)}{v_{2}-v^{3}}+k_{1}\left|v_{2}-v_{4}\right|, \quad k_{1}>0
$$

Since $v_{4}>v_{2}>v^{3}$ and $\left.\sigma\right|_{\alpha\left(u^{3}, v^{3}\right)}>0$, the above inequality gives

$$
\begin{gathered}
g\left(u_{5}, v_{4}\right)-g\left(u^{3}, v^{3}\right)>g\left(u_{2}, v_{2}\right)-g\left(u^{3}, v^{3}\right)+k_{2}\left|v_{2}-v_{4}\right|, \quad k_{2}>0 \\
g\left(u_{5}, v_{4}\right)>g\left(u_{2}, v_{2}\right)+k_{2}\left|v_{2}-v_{4}\right|, \quad k_{2}>0
\end{gathered}
$$

Similarly, (3.4) gives

$$
g\left(u_{2}, v_{2}\right)>g\left(u_{4}, v_{4}\right)+k_{3}\left|v_{2}-v_{4}\right|, \quad k_{3}>0 .
$$

Now (3.7) and (3.8) imply that $g\left(u_{5}, v_{4}\right)>g\left(u_{4}, v_{4}\right)+k\left|v_{2}-v_{4}\right|, k=$ $k_{2}+k_{3}>0$; this contradicts (3.6).

This completes the proof of Theorem 3.2. Q.E.D.

Finally we have the main result of this section.

ThEOREM 3.3. Assume (0.4), (0.5), (2.1), (2.2) and (2.3). There exists at most one solution to any Riemann Problem $\left\{\left(u_{l}, v_{l}\right) ;\left(u_{r}, v_{r}\right)\right\}$ in the class of finite number shocks, rarefaction waves and contact discontinuities; such that condition (E) is satisfied across discontinuities. 
Proof. Suppose the Riemann Problem $\left\{\left(u_{l}, v_{l}\right) ;\left(u_{r}, v_{r}\right)\right\}$ can be solved by connecting $\left(u_{l}, v_{l}\right)$ to $\left(u_{m}, v_{m}\right)$ by backward waves and $\left(u_{m}, v_{m}\right)$ to $\left(u_{r}, v_{r}\right)$ by forward waves; and can also be solved by connecting $\left(u_{l}, v_{l}\right)$ to $\left(\bar{u}_{m}, \bar{v}_{m}\right)$ by backward waves and $\left(\bar{u}_{m}, \bar{v}_{m}\right)$ to $\left(u_{r}, v_{r}\right)$ by forward waves. Then, by Theorem 3.1, we have $\gamma\left(u_{m}, v_{m}\right) \cap \gamma\left(\bar{u}_{m}, \bar{v}_{m}\right) \supset\left\{\left(u_{r}, v_{r}\right)\right\}$ and $\left(u_{m}, v_{m}\right) \in$ $\beta\left(u_{l}, v_{l}\right) ;\left(\bar{u}_{m}, \bar{v}_{m}\right) \in \beta\left(u_{l}, v_{l}\right)$. Thus by Theorem 3.2, $\left(u_{m}, v_{m}\right)=\left(\bar{u}_{m}, \bar{v}_{m}\right)$. Also Theorem 3.1 says that both $\left\{\left(u_{l}, v_{l}\right) ;\left(u_{m}, v_{m}\right)\right\}$ and $\left\{\left(u_{m}, v_{m}\right) ;\left(u_{r}, v_{r}\right)\right\}$ have unique solution. This finishes the proof of Theorem 3.3. Q.E.D.

Combining Theorems 2.3 and 3.3, we finally have the following main theorem.

Theorem. Assume (0.4), (0.5), (2.1), (2.2) and (2.3). There exists a unique solution to any Riemann Problem in the class of shocks, rarefaction waves and contact discontinuities, such that condition $(\mathrm{E})$ is satisfied across discontinuities.

The author wishes to express his gratitude to Professor Joel A. Smoller for his encouragement and also for the inspiration he received from their many stimulating conversations, all of which contributed to the completion of this work.

\section{BIBLIOGRAPHY}

1. J. Glimm, Solution in the large for nonlinear hyperbolic systems of equations, Comm. Pure Appl. Math. 18 (1965), 697-715. MR 33 \#2976.

2. J. A. Smoller and J. L. Johnson, Global solutions for an extended class of hyperbolic systems of conservation laws, Arch. Rational Mech. Anal. 32 (1969), 169-189. MR 38 \#4822.

3. P. D. Lax, Hyperbolic systems of conservation laws. II, Comm. Pure Appl. Math. 10 (1957), 537-566. MR 20 \#176.

4. T. Nishida, Global solution for an initial boundary value problem of a quasilinear hyperbolic system, Proc. Japan Acad. 44 (1968), 642-646. MR 38 \#4821.

5. T. Nishida and J. A. Smoller, Solution in the large for some nonlinear hyperbolic conservation laws, Comm. Pure Appl. Math. 25 (1973), 183-200.

6. O. A. Oleinik, On the uniqueness of the generalized solution of Cauchy problem for nonlinear system of equations occuring in mechanics, Uspehi Mat. Nauk 12 (1957), no. 6 (78), 169-176. (Russian) MR 20 \#1057.

7. J. A. Smoller, On the solution of the Riemann problem with general step data for an extended class of hyperbolic systems, Michigan Math. J. 16 (1969), 201-210. MR 40 \#552.

8. - A uniqueness theorem for Riemann problems, Arch. Rational Mech. Anal. 33 (1969), 110-115. MR 38 \#6238.

DEPARTMENT OF MATHEMATICS, UNIVERSITY OF MARYLAND, COLLEGE PARK, MARYLAND 20742 